KIAS P02049

Saclay T02/102

\title{
Parameter-free effective field theory calculation for the solar proton-fusion and hep processes
}

\author{
T.-S. Park ${ }^{(a, b)}$, L.E. Marcucci ${ }^{(c, d)}$, R. Schiavilla ${ }^{(e, f)}$, M. Viviani ${ }^{(d, c)}$, A. Kievsky ${ }^{(d, c)}$, S. $_{\text {Rosati }}^{(d, c)}$ \\ K. Kubodera ${ }^{(a, b)}$, D.-P. Min $^{(g)}$, and M. Rho ${ }^{(a, h)}$ \\ (a) School of Physics, Korea Institute for Advanced Study, Seoul 130-012, Korea \\ (b) Department of Physics and Astronomy, University of South Carolina, Columbia, SC 29208, USA \\ (c) Department of Physics, University of Pisa, I-56100 Pisa, Italy \\ (d) INFN, Sezione di Pisa, I-56100 Pisa, Italy \\ (e) Department of Physics, Old Dominion University, Norfolk, Virginia 23529, USA \\ (f) Jefferson Lab, Newport News, Virginia 23606, USA \\ (g) Department of Physics, Seoul National University, Seoul 151-742, Korea \\ (h) Service de Physique Théorique, CEA/DSM/SPhT, Unité de recherche associé au CNRS, CEA/Saclay, 91191 \\ Gif-sur-Yvette Cédex, France
}

(October 24, 2018)

\begin{abstract}
Spurred by the recent complete determination of the weak currents in two-nucleon systems up to $\mathcal{O}\left(Q^{3}\right)$ in heavy-baryon chiral perturbation theory, we carry out a parameter-free calculation of the threshold $S$-factors for the solar $p p$ (proton-fusion) and hep processes in an effective field theory that combines the merits of the standard nuclear physics method and systematic chiral expansion. The power of the EFT adopted here is that one can correlate in a unified formalism the weak-current matrix elements of two-, three- and four-nucleon systems. Using the tritium $\beta$-decay rate as an input to fix the only unknown parameter in the theory, we can evaluate the threshold $S$ factors with drastically improved precision; the results are $S_{p p}(0)=3.94 \times(1 \pm 0.004) \times 10^{-25} \mathrm{MeV}$-b and $S_{\text {hep }}(0)=(8.6 \pm 1.3) \times 10^{-20} \mathrm{keV}$-b. The dependence of the calculated $S$-factors on the momentum cutoff parameter $\Lambda$ has been examined for a physically reasonable range of $\Lambda$. This dependence is found to be extremely small for the $p p$ process, and to be within acceptable levels for the hep process, substantiating the consistency of our calculational scheme.
\end{abstract}

PACS number: 12.39.Fe 24.85.+p 26.65.+t

\section{INTRODUCTION}

The standard approach to nuclear physics [1] anchored on wavefunctions obtained from the Schrödinger (or Lippman-Schwinger) equation with "realistic" phenomenological potentials has scored an impressive quantitative success in describing systems with two or more nucleons, achieving in some cases accuracy that defies the existing experimental precision. We refer to this approach as SNPA (standard nuclear physics approach). The advent of quantum chromodynamics (QCD) as the theory of strong interactions raises a logical question: What is the status of SNPA in the context of the fundamental theory, QCD ? Put more bluntly, is SNPA (despite its great success) just a model-dependent approach unrelated to the fundamental theory? In our view this is one of the most important issues in nuclear physics today. In this paper, we propose that SNPA can be properly identified as a legitimate component in the general edifice of QCD. The next important question is: If SNPA is indeed a bona-fide element of QCD, how can we establish an expansion scheme which includes SNPA as an approximation and which allows a systematic calculation of correction terms with error estimation? This systematic correction with an error estimation is not feasible with SNPA alone.

Broadly speaking, formulating nuclear physics calculations starting from effective field theories (EFTs) based on QCD calls for "double-step decimation" [2]. To start with, the "bare" EFT Lagrangian for strong interaction physics in the nonperturbative sector is defined at the chiral scale $\Lambda_{\chi} \sim 4 \pi f_{\pi} \sim 1 \mathrm{GeV}$, with the parameters in the Lagrangian determined by suitably matching them to QCD at that scale [3]. This Lagrangian cannot be directly used for studying complex nuclei. In order to render it applicable to low-energy nuclear physics, it is desirable, if not indispensable, that a "decimation" be made from the chiral scale $\Lambda_{\chi}$ down to what might be called the "Fermi-sea scale" $\Lambda_{F S} \sim k_{F}$, where $k_{F}$ the nuclear Fermi momentum. An effective Lagrangian resulting from the decimation to this Fermi-sea scale is expected to contain parameters that reflect "intrinsic" density-dependence (as suggested in, e.g.,Ref. [4]) in addition to the usual dense loop effects. The next stage of decimation consists of integrating out the effective degrees of freedom and modes from the scale $\Lambda_{F S}$ to $\Lambda_{0} \sim 0$ 
$\mathrm{MeV}$. This stage corresponds to doing shell-model type calculations in finite nuclei [5] and to formulating Fermiliquid theory in nuclear matter [6]. The physics of heavy nuclei or nuclear matter will involve both decimations but, for light nuclei, one can bypass the second decimation and work directly with the chiral Lagrangian.

The aim of this article is to describe a formalism that combines the high accuracy of SNPA and the power of EFT to make totally parameter-free predictions for electroweak transitions in light nuclei. To be concrete, we shall consider the following two solar nuclear fusion processes:

$$
\begin{aligned}
p p: & p+p \rightarrow d+e^{+}+\nu_{e}, \\
\text { hep }: & p+{ }^{3} \mathrm{He} \rightarrow{ }^{4} \mathrm{He}+e^{+}+\nu_{e} .
\end{aligned}
$$

We stress that in our EFT approach these processes involving different numbers of nucleons can be treated on the same footing. A concise account of the present study was previously given in [7] for the $p p$ process, and in [8] for the hep process.

The reactions (11) and (2) figure importantly in astrophysics and particle physics; they have much bearing upon issues of great current interest such as, for example, the solar neutrino problem and non-standard physics in the neutrino sector. Since the thermal energy of the interior of the Sun is of the order of $\mathrm{keV}$, and since no experimental data is available for such low-energy regimes, one must rely on theory for determining the astrophysical $S$-factors of the solar nuclear processes. Here we concentrate on the threshold $S$-factor, $S(0)$, for the reactions (11) and (2). The necessity of a very accurate estimate of the threshold $S$-factor for the $p p$ process, $S_{p p}(0)$, comes from the fact that $p p$ fusion essentially governs the solar burning rate and the vast majority of the solar neutrinos come from this reaction. Meanwhile, the hep process is important in a different context. The hep reaction can produce the highest-energy solar neutrinos with their spectrum extending beyond the maximum energy of the ${ }^{8} \mathrm{~B}$ neutrinos. Therefore, even though the flux of the hep neutrinos is small, there can be, at some level, a significant distortion of the higher end of the ${ }^{8} \mathrm{~B}$ neutrino spectrum due to the hep neutrinos. This change can influence the interpretation of the results of a recent Super-Kamiokande experiment that have generated many controversies related to neutrino oscillations [9, 10]. To address these issues quantitatively, a reliable estimate of $S_{\text {hep }}(0)$ is indispensable.

The primary amplitudes for both the $p p$ and hep processes are of the Gamow-Teller (GT) type $(\Delta J=1$, no parity change). Since the single-particle GT operator is well known at low energy, a major theoretical task is the accurate estimation of the meson-exchange current (MEC) contributions.

The nature of the specific challenge involved here can be elucidated in terms of the chiral filter picture. If the
MEC in a given transition receives an unsuppressed contribution from a one-soft-pion exchange diagram, then we can take advantage of the fact that the soft-pionexchange MEC is uniquely dictated by chiral symmetry 1 and that there is a mechanism (called the chiral filter mechanism) that suppresses higher chiral-order terms 11. 12]. We refer to a transition amplitude to which the chiral filter mechanism is applicable (not applicable) as a chiral-protected (chiral-unprotected) case. It is known that the space component of the vector current and the time component of the axial current are chiralprotected, whereas the time component of the vector current and the space component of the axial current are chiral-unprotected (see Appendix A). This implies among other things that the isovector M1 and axial-charge transitions are chiral-protected [14,15, but that the GT transition is chiral-unprotected. This feature renders the estimation of the GT amplitude a more subtle problem; the physics behind it is that MEC here receives significant short-ranged contributions the strength of which cannot be determined by chiral symmetry alone.

The difficulty becomes particularly pronounced for the hep process for the following reasons. First, the singleparticle GT matrix element for the hep process is strongly suppressed due to the symmetries of the initial and final state wave functions. Secondly, as pointed out in Refs. 16] (referred to as "CRSW91") and 17] (referred to as "SWPC92"), the main two-body corrections to the "leading" one-body GT term tend to come with the opposite sign causing a large cancellation. A recent detailed SNPA calculation by Marcucci et al. [18], hereafter referred to as MSVKRB, has re-confirmed the substantial cancellation between the one-body and two-body terms for the hep GT transition. The two-body terms therefore need to be calculated with great precision, which is a highly non-trivial task. Indeed, an accurate evaluation of the hep rate has been a long-standing challenge in nuclear physics [19]. The degree of this difficulty may be appreciated by noting that theoretical estimates of the hep $S$-factor have varied by orders of magnitude in the literature.

As mentioned, in obtaining accurate estimates of the GT transition amplitudes, it is imperative to have good theoretical control of short-distance physics. We expect that a "first-principle" approach based on effective field theory (EFT) will provide a valuable insight into this issue. We therefore adopt here the approach developed in Refs. [12,20], which purports to combine the highly sophisticated SNPA with an EFT based on chiral dynamics of QCD. Our starting point is the observation that, to high accuracy, the leading-order single-particle

\footnotetext{
${ }^{1} \mathrm{~A}$ more modern and complete discussion on this observation has recently been given by Ananyan, Serot and Walecka [13.
} 
operators in SNPA and EFT are identical, and that their matrix elements can be reliably estimated with the use of realistic SNPA wave functions for the initial and final nuclear states. Next, we note that in EFT the operators representing two-body corrections to the leadingorder one-body term can be controlled by systematic chiral expansion in heavy-baryon chiral perturbation theory $(\mathrm{HB} \chi \mathrm{PT})$ [20]. Then, since the ratio of a two-body matrix element to the leading-order one-body matrix element can be evaluated with sufficient accuracy with the use of the realistic SNPA wave functions 3 , we are in a position to obtain a reliable estimate of the total (onebody + two-body) contribution. This approach takes full advantage of the extreme high accuracy of the wave functions achieved in SNPA while securing a good control of the transition operators via systematic chiral expansion. For convenience, we will refer to this method, which exploits the powers of both SNPA and EFT, as "MEEFT" (short for more effective EFT). MEEFT - which is close in spirit to Weinberg's original scheme [21] based on the chiral expansion of "irreducible terms" - has been found to have an amazing predictive power for the $n+p \rightarrow d+\gamma$ process [14,22] and several other processes [23]. An alternative approach, which however is in line with our reasoning, has been discussed by Ananyan, Serot and Walecka [13].

An early $\mathrm{HB} \chi \mathrm{PT}$ study of the $p p$ process was made in Ref. [24] (hereafter referred to as PKMR98) by four of the authors. The calculation in PKMR98 was carried out up to next-to-next-to-next-to-leading order $\left(\mathrm{N}^{3} \mathrm{LO}\right)$ in chiral counting (see below). At $\mathrm{N}^{3} \mathrm{LO}$, two-body mesonexchange currents (MEC) begin to contribute, and there appears one unknown parameter in the chiral Lagrangian contributing to the MEC. This unknown constant, called $\hat{d}^{R}$ in Ref. 24, represents the strength of a four-nucleonaxial-current contact interaction. In Ref. [24], since no method was known to fix the value of $\hat{d}^{R}$, the $\hat{d}^{R}$-term was simply ignored by invoking a qualitative argument that the short-range repulsive core would strongly suppress its contribution. Due to uncertainties associated with this argument, Ref. [24] was unable to corroborate or exclude the result of the latest SNPA calculation [25], $\delta_{2 \mathrm{~B}}=0.5 \sim 0.8 \%$, where $\delta_{2 \mathrm{~B}}$ is the ratio of the contribution of the two-body MEC to that of the one-body current (see below).

The situation can be greatly improved by using MEEFT. As first discussed in Refs. [7, 8] and as will be ex-

\footnotetext{
${ }^{2}$ The argument made here should apply generally to $n$-body currents $(n \geq 2)$ but since the 2-body terms are dominant, we shall continue to restrict our discussion to the latter.

${ }^{3}$ This statement holds only for the finite-range part of twobody operators, with the zero-range part requiring a regularization to be specified below.
}

pounded here, the crucial point is that exactly the same combination of counter terms that defines the constant $\hat{d}^{R}$ enters into the Gamow-Teller (GT) matrix elements that feature in $p p$ fusion, tritium $\beta$-decay, the hep process, $\mu$-capture on a deuteron, and $\nu-d$ scattering and that the short-range interaction involving the constant $\hat{d}^{R}$ is expected to be "universal," that is, $A$-independent. Therefore, assuming that three- and four-body currents can be ignored (which we will justify a posteriori), if the value of $\hat{d}^{R}$ can be fixed using one of the above processes, then we can make a totally parameter-free prediction for the GT matrix elements of the other processes. Indeed, the existence of accurate experimental data for the tritium $\beta$-decay rate, $\Gamma_{\beta}^{t}$, and the availability of extremely well tested realistic wave functions for the $A=3$ nuclear systems allow us to carry out this program. In the present work we determine the value of $\hat{d}^{R}$ from $\Gamma_{\beta}^{t}$ and perform parameter-free EFT-based calculations of $S_{p p}(0)$ and $S_{\text {hep }}(0)$.

As described below, our scheme has a cutoff parameter $\Lambda$, which defines the energy/momentum cutoff scale of EFT below which reside the chosen explicit degrees of freedomf. Obviously, in order for our result to be physically acceptable, its cutoff dependence must be under control. In our scheme, for a given value of $\Lambda$ in a physically reasonable range (to be discussed later), $\hat{d}^{R}$ is determined to reproduce $\Gamma_{\beta}^{t}$; thus $\hat{d}^{R}$ is a function of $\Lambda$. According to the premise of EFT, even if $\hat{d}^{R}$ itself is $\Lambda$-dependent, physical observables (in our case the $S$-factors) should be independent of $\Lambda$ as required by renormalization-group invariance. We shall show that our results meet this requirement to a satisfactory degree. The robustness of our calculational results against changes in $\Lambda$ allows us to make predictions on $S_{p p}(0)$ and $S_{\text {hep }}(0)$ with much higher precision than hitherto achieved. Thus we predict: $S_{p p}(0)=3.94 \times(1 \pm 0.004) \times$ $10^{-25} \mathrm{MeV}-\mathrm{b}$ and $S_{\text {hep }}(0)=(8.6 \pm 1.3) \times 10^{-20} \mathrm{keV}-\mathrm{b}$.

The remainder of this article is organized as follows. In Section II we briefly explain our formalism; in particular, we describe the relevant transition operators derived in $\mathrm{HB} \chi \mathrm{PT}$. Section III presents the calculation of $S_{p p}(0)$, while Section IV is concerned with the estimation of $S_{\text {hep }}(0)$. Section V is devoted to discussion and conclusions. We have made the explanation of the formalism in the text as brief and focused as possible, relegating most technical details to the Appendices.

\footnotetext{
${ }^{4}$ The cutoff specifies not just the relevant degrees of freedom but also their momentum/energy content. This should be understood in what follows although we do not always mention it.
} 


\section{FORMALISM}

We sketch here the basic elements of our formalism. The explicit degrees of freedom taken into account in our scheme are the nucleon and the pion, with all other degrees of freedom ( $\rho$ - and $\omega$-mesons, $\Delta(1232)$, etc.) integrated out. The $\mathrm{HB} \chi \mathrm{PT}$ Lagrangian can be written as

$$
\mathcal{L}=\sum_{\lambda} \mathcal{L}_{\lambda}=\mathcal{L}_{0}+\mathcal{L}_{1}+\cdots
$$

with the chiral order $\lambda$ defined as

$$
\lambda \equiv d+e+\frac{n}{2}-2
$$

where $d, e$ and $n$ are, respectively, the numbers of derivatives (the pion mass counted as one derivative), external fields and nucleon lines belonging to a vertex. Chiral symmetry requires $\lambda \geq 0$. The leading-order Lagrangian is given by

$$
\begin{aligned}
\mathcal{L}_{0} & =\bar{B}\left[i v \cdot D+2 i g_{A} S \cdot \Delta\right] B-\frac{1}{2} \sum_{A} C_{A}\left(\bar{B} \Gamma_{A} B\right)^{2} \\
& +f_{\pi}^{2} \operatorname{Tr}\left(i \Delta^{\mu} i \Delta_{\mu}\right)+\frac{f_{\pi}^{2}}{4} \operatorname{Tr}\left(\chi_{+}\right),
\end{aligned}
$$

where $B$ is the nucleon field in $\mathrm{HB} \chi \mathrm{PT} ; g_{A}=1.2670 \pm$ 0.0035 is the axial-vector coupling constant [28], and $f_{\pi}=92.4 \mathrm{MeV}$ is the pion decay constant. Furthermore

$$
\begin{aligned}
D_{\mu} B & =\left(\partial_{\mu}+\Gamma_{\mu}\right) B, \\
\Gamma_{\mu} & =\frac{1}{2}\left[\xi^{\dagger}, \partial_{\mu} \xi\right]-\frac{i}{2} \xi^{\dagger} R_{\mu} \xi-\frac{i}{2} \xi L_{\mu} \xi^{\dagger}, \\
\Delta_{\mu} & =\frac{1}{2}\left[\xi^{\dagger}, \partial_{\mu} \xi\right]+\frac{i}{2} \xi^{\dagger} R_{\mu} \xi-\frac{i}{2} \xi L_{\mu} \xi^{\dagger}, \\
\chi_{+} & =\xi^{\dagger} \chi \xi^{\dagger}+\xi \chi^{\dagger} \xi
\end{aligned}
$$

with

$$
\xi=\sqrt{\Sigma}=\exp \left(i \frac{\vec{\tau} \cdot \vec{\pi}}{2 f_{\pi}}\right) .
$$

$R_{\mu} \equiv \frac{\tau^{a}}{2}\left(\mathcal{V}_{\mu}^{a}+\mathcal{A}_{\nu}^{a}\right)$ and $L_{\mu}=\frac{\tau^{a}}{2}\left(\mathcal{V}_{\mu}^{a}-\mathcal{A}_{\nu}^{a}\right)$ denote external gauge fields, and $\chi$ is proportional to the quark mass matrix. If we neglect the small isospin-symmetry breaking, then $\chi=m_{\pi}^{2}$ (in the absence of external scalar and pseudo-scalar fields). For convenience, we work in the reference frame in which the four-velocity $v^{\mu}$ and the spin operator $S^{\mu}$ are

$$
v^{\mu}=(1, \mathbf{0}) \text { and } S^{\mu}=\left(0, \frac{\boldsymbol{\sigma}}{2}\right) .
$$

The NLO Lagrangian (the so-called " $1 / m$ " term) in the one-nucleon sector is given in Ref. [26], while that in the two-nucleon sector is given in Ref. [27 $]^{5}$. With fourfermion contact terms included, the Lagrangian takes the form

$$
\begin{aligned}
\mathcal{L}_{1}= & \bar{B}\left\{\frac{v^{\mu} v^{\nu}-g^{\mu \nu}}{2 m_{N}} D_{\mu} D_{\nu}+4 c_{3} i \Delta \cdot i \Delta\right. \\
& +\left(2 c_{4}+\frac{1}{2 m_{N}}\right)\left[S^{\mu}, S^{\nu}\right]\left[i \Delta_{\nu}, i \Delta_{\nu}\right] \\
& \left.-i \frac{1+c_{6}}{m_{N}}\left[S^{\mu}, S^{\nu}\right] f_{\mu \nu}^{+}\right\} B-4 i d_{1} \bar{B} S \cdot \Delta B \bar{B} B \\
& +2 i d_{2} \epsilon^{a b c} \epsilon_{\mu \nu \lambda \delta} v^{\mu} \Delta^{\nu, a} \bar{B} S^{\lambda} \tau^{b} B \bar{B} S^{\delta} \tau^{c} B \\
& +\cdots,
\end{aligned}
$$

where $m_{N} \simeq 939 \mathrm{MeV}$ is the nucleon mass, and

$$
\begin{aligned}
f_{\mu \nu}^{+}=\xi & \left(\partial_{\mu} L_{\nu}-\partial_{\nu} L_{\mu}-i\left[L_{\mu}, L_{\nu}\right]\right) \xi^{\dagger} \\
& +\xi^{\dagger}\left(\partial_{\mu} R_{\nu}-\partial_{\nu} R_{\mu}-i\left[R_{\mu}, R_{\nu}\right]\right) \xi
\end{aligned}
$$

$\epsilon_{0123}=1$, and $\Delta_{\mu}=\frac{\tau^{a}}{2} \Delta_{\mu}^{a}$. We have shown here only those terms which are directly relevant to our present study. The dimensionless low-energy-constants (LECs), $\hat{c}$ 's and $\hat{d}$ 's, are defined as

$$
c_{3,4}=\frac{1}{m_{N}} \hat{c}_{3,4}, \quad d_{1,2}=\frac{g_{A}}{m_{N} f_{\pi}^{2}} \hat{d}_{1,2} .
$$

We now consider the chiral counting of the electroweak currents (see the Appendices for details). In the present scheme it is sufficient to focus on "irreducible graphs" in Weinberg's classification [21]. Irreducible graphs are organized according the chiral index $\nu$ given by

$$
\nu=2(A-C)+2 L+\sum_{i} \nu_{i},
$$

where $A$ is the number of nucleons involved in the process, $C$ the number of disconnected parts, and $L$ the number of loops; $\nu_{i}$ is the chiral index $\lambda$, eq.(位), of the $i$-th vertex. One can show that a diagram characterized by eq. (12) involves an $n_{B}$-body transition operator, where $n_{B} \equiv A-C+1$. The physical amplitude is expanded with respect to $\nu$. As explained at length in the Appendix, the leading-order one-body GT operator belongs to $\nu=0$. Compared with this operator, a Feynman diagram with a chiral index $\nu$ is suppressed by a factor of $\left(Q / \Lambda_{\chi}\right)^{\nu}$, where $Q$ is a typical three-momentum scale or the pion mass, and $\Lambda_{\chi} \sim 1 \mathrm{GeV}$ is the chiral scale. In $^{\circ}$

\footnotetext{
${ }^{5}$ Our definition of the pion field here is different from that used in Ref. 27]; we have changed the sign of the pion field. Furthermore, we employ here manifestly Lorentz-invariant and chiral-invariant interactions.

${ }^{6}$ For convenience, a chiral order corresponding to $\nu$ is often referred to as $\mathrm{N}^{\nu} \mathrm{LO} ; \nu=1$ corresponds to NLO (next-toleading order), $\nu=2$ to $\mathrm{N}^{2} \mathrm{LO}$ (next-to-next-to-leading order), and so on.
} 
our case it is important to take into account the kinematic suppression of the time component of the nucleon four-momentum. We note

$$
v \cdot p_{l} \sim v \cdot p_{l}^{\prime} \sim v \cdot k_{l} \sim \frac{Q^{2}}{m_{N}},
$$

where $p_{l}^{\mu}\left(p_{l}^{\prime \mu}\right)$ denotes the initial (final) momentum of the $l$-th nucleon, and $k_{l}^{\mu} \equiv\left(p_{l}^{\prime}-p_{l}\right)^{\mu}$. Therefore, each appearance of $v \cdot p_{l}, v \cdot p_{l}^{\prime}$ or $v \cdot k_{l}$ carries two powers of $Q$ instead of one, which implies that $\nu$ increases by two units rather than one. It is also to be noted that, if we denote by $q^{\mu}=\left(q_{0}, \boldsymbol{q}\right)$ the momentum transferred to the leptonic pair in eqs.(11) (2), then $q_{0} \sim|\boldsymbol{q}| \sim Q^{2} / \Lambda_{\chi}$ $\sim \mathcal{O}\left(Q^{2}\right)$ rather than $\mathcal{O}(Q)$ as naive counting would suggest. These features turn out to simplify our calculation considerably.

In this paper, as far as the main calculation is concerned, we shall limit ourselves to $\mathrm{N}^{3} \mathrm{LO}$; for certain discussions, however, we shall consider operators belonging to $\mathrm{N}^{4} \mathrm{LO}$ as well.

We now describe the derivation of one-body (1B) and two-body (2B) current operators with due consideration of chiral counting. The current in momentum space is written as

$$
J^{\mu}(\boldsymbol{q})=V^{\mu}(\boldsymbol{q})+A^{\mu}(\boldsymbol{q})=\int d \boldsymbol{x} \mathrm{e}^{-i \boldsymbol{q} \cdot \boldsymbol{x}} J^{\mu}(\boldsymbol{x}) .
$$

When necessity arises to distinguish the space and time components of the currents, we use the notations

$$
V^{\mu}=\left(V^{0}, \boldsymbol{V}\right), \quad A^{\mu}=\left(A^{0}, \boldsymbol{A}\right) .
$$

For the clarity of presentation, we first give a summary chart of the basic chiral counting characteristics of the relevant currents, and then provide more detailed explanations in the remainder of this section and in the Appendices. The chiral counting of the electroweak currents is summarized in Table If, where the non-vanishing contributions at $\boldsymbol{q}=0$ are indicated.7

We now discuss the entries of this table order by order:

- $\nu=0$ : One-body $\boldsymbol{A}$ and $V^{0}: \boldsymbol{A}$ gives the GamowTeller (GT) operator, while $V^{0}$ is responsible for the charge operator.

- $\nu=1$ : One-body $A^{0}$ and $\boldsymbol{V}: A^{0}$ gives the axialcharge operator while $\boldsymbol{V}$ gives the M1 operator.

\footnotetext{
${ }^{7}$ For small but finite values of $\boldsymbol{q} \neq 0$, there are slight deviations from this chart; for instance, for all the four cases in the table, there arise one-body contributions at NLO and at higher orders. However, these deviations are not significant in our case, which involves very small values of $\boldsymbol{q}$.
}

TABLE I. Contributions from each type of current at $\boldsymbol{q}=0$. The entry of "-" indicates the absence of contribution. "1B-RC" stands for relativistic corrections to the one-body operators, and "2B-1L" for one-loop 2-body contributions including counter term contributions.

\begin{tabular}{|l||l|l|l|l|l|}
\hline \hline$J^{\mu}$ & $\mathrm{LO}$ & $\mathrm{NLO}$ & $\mathrm{N}^{2} \mathrm{LO}$ & $\mathrm{N}^{3} \mathrm{LO}$ & $\mathrm{N}^{4} \mathrm{LO}$ \\
\hline $\boldsymbol{A}$ & $1 \mathrm{~B}$ & - & $1 \mathrm{~B}-\mathrm{RC}$ & $2 \mathrm{~B}$ & $1 \mathrm{~B}-\mathrm{RC}, 2 \mathrm{~B}-1 \mathrm{~L}$ and 3B \\
$A^{0}$ & - & $1 \mathrm{~B}$ & $2 \mathrm{~B}$ & $1 \mathrm{~B}-\mathrm{RC}$ & $1 \mathrm{~B}-\mathrm{RC}, 2 \mathrm{~B}-1 \mathrm{~L}$ \\
$\boldsymbol{V}$ & - & $1 \mathrm{~B}$ & $2 \mathrm{~B}$ & $1 \mathrm{~B}-\mathrm{RC}$ & $1 \mathrm{~B}-\mathrm{RC}, 2 \mathrm{~B}-1 \mathrm{~L}$ \\
$V^{0}$ & $1 \mathrm{~B}$ & - & - & $2 \mathrm{~B}$ & $1 \mathrm{~B}-\mathrm{RC}, 2 \mathrm{~B}-1 \mathrm{~L}$ and 3B \\
\hline \hline
\end{tabular}

- $\nu=2$ : Two-body tree current with $\nu_{i}=0$ vertices, namely, the soft-pion-exchange current. This is a leading correction to the one-body M1 and axialcharge operators carrying an odd orbital angular momentum.

- $\nu=3$ : Two-body tree currents with $\sum_{i} \nu_{i}=1$, which correspond to the hard-pion current, considered in CRSW91 [16] and SWPC92 [17]. These are leading corrections to the GT and $V^{0}$ operators carrying an even orbital angular momentum.

- $\nu=4$ : All the components of the electroweak current receive contributions of this order. They consist of two-body one-loop corrections as well as leading-order (tree) three-body corrections. Among the three-body currents, however, there are no six-fermion contact terms proportional to $(\bar{N} N)^{3}$, because there is no derivative at the vertex and hence no external field.

It is noteworthy that the counting rule for $\boldsymbol{V}$ is the same as for $A^{0}$, and the counting rules for $V^{0}$ and $\boldsymbol{A}$ are the same. The behavior of $\boldsymbol{V}$ and $A^{0}$ summarized in Table If represents the chiral filter mechanism [1], and $\boldsymbol{V}$ and $A^{0}$ are referred to as chiral-filter-protected currents. By contrast, $V^{0}$ and $\boldsymbol{A}$ belong to chiral-filter-unprotected currents.

We now discuss the explicit expressions for the relevant currents. For the one-body (1B) currents, for both the vector and axial cases, one can simply carry over the expressions obtained in MSVKRB. Up to $\mathrm{N}^{3} \mathrm{LO}$, the $1 \mathrm{~B}$ currents in coordinate representaion are given as

$$
\begin{aligned}
\tilde{V}^{0}(l)= & \tau_{l}^{-} \mathrm{e}^{-i \boldsymbol{q} \cdot \boldsymbol{r}_{l}}\left[1+i \boldsymbol{q} \cdot \boldsymbol{\sigma}_{l} \times \boldsymbol{p}_{l} \frac{2 \mu_{V}-1}{4 m_{N}^{2}}\right] \\
\tilde{\boldsymbol{V}}(l)= & \tau_{l}^{-} \mathrm{e}^{-i \boldsymbol{q} \cdot \boldsymbol{r}_{l}}\left[\frac{\overline{\boldsymbol{p}}_{l}}{m_{N}}\left(1-\frac{\overline{\boldsymbol{p}}_{l}^{2}}{2 m_{N}^{2}}\right)+i \frac{\mu_{V}}{2 m_{N}} \boldsymbol{q} \times \boldsymbol{\sigma}_{l}\right. \\
& \left.+i \boldsymbol{\sigma}_{l} \times \overline{\boldsymbol{p}}_{l} q_{0} \frac{2 \mu_{V}-1}{4 m_{N}^{2}}\right] \\
\tilde{A}^{0}(l)= & -g_{A} \tau_{l}^{-} \mathrm{e}^{-i \boldsymbol{q} \cdot \boldsymbol{r}_{l}}\left[\frac{\boldsymbol{\sigma}_{l} \cdot \overline{\boldsymbol{p}}_{l}}{m_{N}}\left(1-\frac{\overline{\boldsymbol{p}}_{l}^{2}}{2 m_{N}^{2}}\right)\right] \\
\tilde{\boldsymbol{A}}(l)= & -g_{A} \tau_{l}^{-} \mathrm{e}^{-i \boldsymbol{q} \cdot \boldsymbol{r}_{l}}\left[\boldsymbol{\sigma}_{l}\right.
\end{aligned}
$$




$$
\left.+\frac{2\left(\overline{\boldsymbol{p}}_{l} \boldsymbol{\sigma}_{l} \cdot \overline{\boldsymbol{p}}_{l}-\boldsymbol{\sigma}_{l} \overline{\boldsymbol{p}}_{l}^{2}\right)+i \boldsymbol{q} \times \overline{\boldsymbol{p}}_{l}}{4 m_{N}^{2}}\right],
$$

where $\mu_{V} \simeq 4.70$ is the isovector anomalous magnetic moment of the nucleon. Eq.16) gives the isospinlowering currents,

$$
J_{\mu} \equiv J_{\mu}^{a=1}-i J_{\mu}^{a=2},
$$

and $\tau_{l}^{-} \equiv \frac{1}{2}\left(\tau_{l}^{x}-i \tau_{l}^{y}\right)$. The tildes in eq.(16) imply that tation, and $\boldsymbol{p}_{l}=-i \nabla_{l}$ and $\overline{\boldsymbol{p}}_{l}=-\frac{i}{2}\left(\vec{\nabla}_{l}-\overleftarrow{\nabla}_{l}\right)$ act on the wave functions.

We next discuss the two-body (2B) currents. The expressions for the $\boldsymbol{V}_{2 \mathrm{~B}}$ and $A_{2 \mathrm{~B}}^{0}$ operators can be found in [20,50]. The $V_{2 \mathrm{~B}}^{0}$ operator does not appear up to the order under consideration. The derivation of the $2 \mathrm{~B}$ axial current, $\boldsymbol{A}_{2 \mathrm{~B}}$, in $\mathrm{HB} \chi \mathrm{PT}$ is described in Appendix A. In momentum space, $\boldsymbol{A}_{2 \mathrm{~B}}$ reads

$$
\begin{aligned}
\boldsymbol{A}_{2 \mathrm{~B}}= & \sum_{l<m}^{A} \boldsymbol{A}_{l m}, \\
\boldsymbol{A}_{12}= & \frac{g_{A}}{2 m_{N} f_{\pi}^{2}} \frac{1}{m_{\pi}^{2}+\boldsymbol{k}^{2}}\left[-\frac{i}{2} \tau_{\times}^{-} \boldsymbol{p}\left(\boldsymbol{\sigma}_{1}-\boldsymbol{\sigma}_{2}\right) \cdot \boldsymbol{k}\right. \\
& +4 \hat{c}_{3} \boldsymbol{k} \boldsymbol{k} \cdot\left(\tau_{1}^{-} \boldsymbol{\sigma}_{1}+\tau_{2}^{-} \boldsymbol{\sigma}_{2}\right) \\
& \left.+\left(\hat{c}_{4}+\frac{1}{4}\right) \tau_{\times}^{-} \boldsymbol{k} \times\left[\boldsymbol{\sigma}_{\times} \times \boldsymbol{k}\right]\right] \\
& -\frac{g_{A}}{m_{N} f_{\pi}^{2}}\left[2 \hat{d}_{1}\left(\tau_{1}^{-} \boldsymbol{\sigma}_{1}+\tau_{2}^{-} \boldsymbol{\sigma}_{2}\right)+\hat{d}_{2} \tau_{\times}^{a} \boldsymbol{\sigma}_{\times}\right],
\end{aligned}
$$
$\left.i \tau_{l}^{y}\right), \tau_{\times}^{a} \equiv\left(\tau_{1} \times \tau_{2}\right)^{x}-i\left(\tau_{1} \times \tau_{2}\right)^{y}$, and similarly for $\boldsymbol{\sigma}_{\times}$; $\hat{c}$ 's and $\hat{d}$ 's are the LECs explained in PKMR98. The values of $\hat{c}$ 's in Eq. 18 have been determined from $\pi$ $N$ data [29]: $\hat{c}_{3}=-3.66 \pm 0.08$ and $\hat{c}_{4}=2.11 \pm 0.08$. The two constants, $\hat{d}_{1}$ and $\hat{d}_{2}$, remain to be fixed but it turns out (see Appendix C.2) that, thanks to Fermi-Dirac statistics, only one combination of them,

$$
\hat{d}^{R} \equiv \hat{d}_{1}+2 \hat{d}_{2}+\frac{1}{3} \hat{c}_{3}+\frac{2}{3} \hat{c}_{4}+\frac{1}{6}
$$

is relevant in the present context. 8

It should be noted that the two-body currents given in eqs. (18) are valid only up to a certain cutoff $\Lambda$. This implies that, when we go to coordinate space, the currents must be regulated. This is a key point in our approach. Specifically, in performing Fourier transformation to derive the $r$-space representation of a transition operator, the currents are given in the coordinate space represen-

with $\boldsymbol{p} \equiv\left(\overline{\boldsymbol{p}}_{1}-\overline{\boldsymbol{p}}_{2}\right) / 2, \overline{\boldsymbol{p}}_{l} \equiv\left(\boldsymbol{p}_{l}+\boldsymbol{p}_{l}^{\prime}\right) / 2, \tau_{l}^{-} \equiv \frac{1}{2}\left(\tau_{l}^{x}-\right.$

we use the Gaussian regularization (see Appendix C). This is, to good accuracy, equivalent to replacing the delta and Yukawa functions with the corresponding regulated functions,

$$
\begin{aligned}
\delta_{\Lambda}^{(3)}(r) & \equiv \int \frac{d^{3} \boldsymbol{k}}{(2 \pi)^{3}} S_{\Lambda}^{2}\left(\boldsymbol{k}^{2}\right) \mathrm{e}^{i \boldsymbol{k} \cdot \boldsymbol{r}}, \\
y_{0 \Lambda}^{\pi}(r) & \equiv \int \frac{d^{3} \boldsymbol{k}}{(2 \pi)^{3}} S_{\Lambda}^{2}\left(\boldsymbol{k}^{2}\right) \mathrm{e}^{i \boldsymbol{k} \cdot \boldsymbol{r}} \frac{1}{\boldsymbol{k}^{2}+m_{\pi}^{2}} \\
y_{1 \Lambda}^{\pi}(r) & \equiv-r \frac{\partial}{\partial r} y_{0 \Lambda}^{\pi}(r) \\
y_{2 \Lambda}^{\pi}(r) & \equiv \frac{1}{m_{\pi}^{2}} r \frac{\partial}{\partial r} \frac{1}{r} \frac{\partial}{\partial r} y_{0 \Lambda}^{\pi}(r),
\end{aligned}
$$

where the cut-off function, $S_{\Lambda}\left(\boldsymbol{k}^{2}\right)$, is defined as

$$
S_{\Lambda}\left(\boldsymbol{k}^{2}\right)=\exp \left(-\frac{\boldsymbol{k}^{2}}{2 \Lambda^{2}}\right) \text {. }
$$

The resulting $r$-space expressions of the currents in the center-of-mass (c.m.) frame are

$$
\begin{aligned}
\boldsymbol{V}_{12}(\boldsymbol{r})= & -\frac{g_{A}^{2} m_{\pi}^{2}}{12 f_{\pi}^{2}} \tau_{\times}^{-} \boldsymbol{r}\left[\boldsymbol{\sigma}_{1} \cdot \boldsymbol{\sigma}_{2} y_{0 \Lambda}^{\pi}(r)+S_{12} y_{2 \Lambda}^{\pi}(r)\right] \\
- & i \frac{g_{A}^{2}}{8 f_{\pi}^{2}} \boldsymbol{q} \times\left[\mathcal{O}_{\times} y_{0 \Lambda}^{\pi}(r)+\left(\mathcal{T}_{\times}-\frac{2}{3} \mathcal{O}_{\times}\right) y_{1 \Lambda}^{\pi}(r)\right], \\
A_{12}^{0}(\boldsymbol{r})= & -\frac{g_{A}}{4 f_{\pi}^{2}} \tau_{\times}^{-}\left[\frac{\boldsymbol{\sigma}_{+} \cdot \hat{\boldsymbol{r}}}{r}+\frac{i}{2} \boldsymbol{q} \cdot \hat{\boldsymbol{r}} \boldsymbol{\sigma}_{-} \cdot \hat{\boldsymbol{r}}\right] y_{1 \Lambda}^{\pi}(r), \\
\boldsymbol{A}_{12}(\boldsymbol{r})= & -\frac{g_{A} m_{\pi}^{2}}{2 m_{N} f_{\pi}^{2}}[ \\
& {\left[\frac{\hat{c}_{3}}{3}\left(\mathcal{O}_{+}+\mathcal{O}_{-}\right)+\frac{2}{3}\left(\hat{c}_{4}+\frac{1}{4}\right) \mathcal{O}_{\times}\right] y_{0 \Lambda}^{\pi}(r) } \\
& \left.+\left[\hat{c}_{3}\left(\mathcal{T}_{+}+\mathcal{T}_{-}\right)-\left(\hat{c}_{4}+\frac{1}{4}\right) \mathcal{T}_{\times}\right] y_{2 \Lambda}^{\pi}(r)\right] \\
+ & \frac{g_{A}}{2 m_{N} f_{\pi}^{2}}\left[\frac{1}{2} \tau_{\times}^{-}\left(\overline{\boldsymbol{p}}_{1} \boldsymbol{\sigma}_{2} \cdot \hat{\boldsymbol{r}}_{+} \overline{\boldsymbol{p}}_{2} \boldsymbol{\sigma}_{1} \cdot \hat{\boldsymbol{r}}\right) \frac{y_{1 \Lambda}^{\pi}(r)}{r}\right. \\
& \left.+\delta_{\Lambda}(r) \hat{d}^{R} \mathcal{O}_{\times}\right],
\end{aligned}
$$

where $\boldsymbol{r}=\boldsymbol{r}_{1}-\boldsymbol{r}_{2}, S_{12}=3 \boldsymbol{\sigma}_{1} \cdot \hat{\boldsymbol{r}} \boldsymbol{\sigma}_{2} \cdot \hat{\boldsymbol{r}}-\boldsymbol{\sigma}_{1} \cdot \boldsymbol{\sigma}_{2}$, and $\mathcal{O}_{\odot}^{k} \equiv \tau_{\odot}^{-} \sigma_{\odot}^{k}, \mathcal{O}_{\odot} \equiv \tau_{\odot}^{-} \boldsymbol{\sigma}_{\odot}, \mathcal{T}_{\odot} \equiv \hat{\boldsymbol{r}} \hat{\boldsymbol{r}} \cdot \mathcal{O}_{\odot}-\frac{1}{3} \mathcal{O}_{\odot}$, $\odot= \pm, \times, \tau_{\odot}^{-} \equiv\left(\tau_{1} \odot \tau_{2}\right)^{-} \equiv\left(\tau_{1} \odot \tau_{2}\right)^{x}-i\left(\tau_{1} \odot \tau_{2}\right)^{y}$ and $\boldsymbol{\sigma}_{\odot} \equiv\left(\boldsymbol{\sigma}_{1} \odot \boldsymbol{\sigma}_{2}\right)$. We emphasize again that $\boldsymbol{A}_{12}$ in Eq. (22) contains only one unknown LEC, $\hat{d}^{R}$, that needs to be fixed using an empirical input. As mentioned in Section I, we choose here to determine $\hat{d}^{R}$ using the experimental value of $\Gamma_{\beta}^{t}$.

\section{DETERMINATION OF $\hat{d}^{R}$}

${ }^{8}$ A sign error made in the expression for $\hat{d}^{R}$ in PKMR98 has been corrected here.
The cutoff parameter $\Lambda$ characterizes the energymomentum scale of our EFT. A reasonable range of $\Lambda$ 
may be inferred as follows. According to the general tenet of $\chi \mathrm{PT}, \Lambda$ larger than $\Lambda_{\chi} \simeq 4 \pi f_{\pi} \simeq m_{N}$ has no physical meaning. Meanwhile, since the pion is an explicit degree of freedom in our scheme, $\Lambda$ should be much larger than the pion mass to ascertain that genuine low-energy contributions are properly included. These considerations lead us to adopt $\Lambda=500-800 \mathrm{MeV}$ as a natural range.

In the present work we use as representative values $\Lambda$ $=500,600$ and $800 \mathrm{MeV}$, and for each of these values of $\Lambda$ we adjust $\hat{d}^{R}$ to reproduce the experimental value of $\Gamma_{\beta}^{t}$. With the use of the value of $\hat{d}^{R}$ so determined, we evaluate the $p p$ and the hep amplitudes. If

To determine $\hat{d}^{R}$ from $\Gamma_{\beta}^{t}$, we calculate $\Gamma_{\beta}^{t}$ from the matrix elements of the current operators evaluated for accurate $A=3$ nuclear wave functions. We employ here the wave functions obtained in Refs. [18,30 using the correlated-hyperspherical-harmonics $(\mathrm{CHH})$ method [31,32]. It is obviously important to maintain consistency between the treatments of the $A=2,3$ and 4 systems. We shall use here the same Argonne $v_{18}$ (AV18) potential [33] for all these nuclei. For the $A \geq 3$ systems we add the Urbana-IX (AV18/UIX) three-nucleon potential [34]. Furthermore, we apply the same regularization method to all the systems in order to control short-range physics in a consistent manner.

The values of $\hat{d}^{R}$ determined in this manner are:

$$
\begin{array}{ll}
\hat{d}^{R}=1.00 \pm 0.07 & \text { for } \Lambda=500 \mathrm{MeV}, \\
\hat{d}^{R}=1.78 \pm 0.08 & \text { for } \Lambda=600 \mathrm{MeV}, \\
\hat{d}^{R}=3.10 \pm 0.10 & \text { for } \Lambda=800 \mathrm{MeV},
\end{array}
$$

where the errors correspond to the experimental uncertainty in $\Gamma_{\beta}^{t}$. Once $\hat{d}^{R}$ has been determined, we are in a position to make a parameter-free calculation of the transition amplitudes for $p p$ and hep, which will be described in the next two sections.

\section{THE $p p$ PROCESS}

It is convenient to decompose the matrix element of the GT operator into one-body and two-body parts

$$
\mathcal{M}=\mathcal{M}_{1 \mathrm{~B}}+\mathcal{M}_{2 \mathrm{~B}} .
$$

We discuss them separately. In PKMR98, an extensive analysis was made of the leading-order (LO) one-body

\footnotetext{
${ }^{9}$ The masses of the light-quark vector mesons $(\rho$ and $\omega)$ are less than $800 \mathrm{MeV}$; therefore, with the use of $\Lambda=800 \mathrm{MeV}$, an accurate description of certain observables might require the explicit presence of the vector mesons, even though the probe energy/momentum in question is much smaller than $800 \mathrm{MeV}$. Thus the results with the cutoff $\Lambda=800 \mathrm{MeV}$ should be taken with some caution.
}

matrix element $\mathcal{M}_{1 \mathrm{~B}}^{C+N}$, with a focus on the connection between EFT and the effective range expansion. The results obtained with the AV18 potential [33 were

$$
\begin{aligned}
\mathcal{M}_{1 \mathrm{~B}}^{C+N}= & (1 \mp 0.02 \% \mp 0.07 \% \mp 0.02 \%) \\
& \times 4.859 \mathrm{fm},
\end{aligned}
$$

where the errors are due to uncertainties in the scattering length and effective ranges. The "full" one-body contribution in PKMR98 includes the vacuum-polarization $(\mathrm{VP})$ and two-photon-exchange $(\mathrm{C} 2)$ contributions. In PKMR98, however, the one-body current due to the $1 / m_{N}^{2}$ term in eq. A.3) was ignored. Although this term is required for formal consistency, its numerical value turns out to be quite small, $\mathcal{M}_{1 \mathrm{~B}}^{1 / m_{N}^{2}}=-0.006 \mathrm{fm}$. In terms of the $\Lambda_{p p}$ defined in Ref. [35].10 we have

$$
\Lambda_{p p}^{2} \equiv \frac{\left|a^{C}\right|^{2} \gamma^{3}}{2} A_{S}^{2} \mathcal{M}_{1 \mathrm{~B}}^{2}=6.91
$$

for the central value, where $a^{C}$ is the $p p{ }^{1} \mathrm{~S}_{0}$ scattering length, and $\gamma$ and $A_{S}$ are the wave number and S-wave normalization constant pertinent to the deuteron, respectively. This should be compared with 6.93 obtained in Ref. [24].

The properly regularized two-body GT matrix elements for the $p p$ process read

$$
\begin{aligned}
\mathcal{M}_{2 \mathrm{~B}}= & \frac{2}{m_{N} f_{\pi}^{2}} \int_{0}^{\infty} d r\{ \\
& \frac{m_{\pi}^{2}}{3}\left(\hat{c}_{3}+2 \hat{c}_{4}+\frac{1}{2}\right) y_{0 \Lambda}^{\pi}(r) u_{d}(r) u_{p p}(r) \\
- & \sqrt{2} \frac{m_{\pi}^{2}}{3}\left(\hat{c}_{3}-\hat{c}_{4}-\frac{1}{4}\right) y_{2 \Lambda}^{\pi}(r) w_{d}(r) u_{p p}(r) \\
+ & \frac{y_{1 \Lambda}^{\pi}(r)}{12 r}\left[\left[u_{d}(r)-\sqrt{2} w_{d}(r)\right] u_{p p}^{\prime}(r)\right. \\
- & {\left.\left[u_{d}^{\prime}(r)-\sqrt{2} w_{d}^{\prime}(r)\right] u_{p p}(r)+\frac{3 \sqrt{2}}{r} w_{d}(r) u_{p p}(r)\right] } \\
- & \left.\hat{d}^{R} \delta_{\Lambda}^{(3)}(r) u_{d}(r) u_{p p}(r)\right\},
\end{aligned}
$$

where $u_{d}(r)$ and $w_{d}(r)$ are the S- and D-wave components of the deuteron wave function, and $u_{p p}(r)$ is the ${ }^{1} \mathrm{~S}_{0} p p$ scattering wave (at zero relative energy). The results are given for the three representative values of $\Lambda$ in Table II; for convenience, the values of $\hat{d}^{R}$ given in Eq.(23) are also listed. The table indicates that, although the value of $\hat{d}^{R}$ is sensitive to $\Lambda, \mathcal{M}_{2 \mathrm{~B}}$ is amazingly stable against the variation of $\Lambda$ within the stated range. In view of this

\footnotetext{
${ }^{10}$ The subscript $p p$ has been added here to avoid confusion with the cutoff parameter $\Lambda$.
} 
high stability, we believe that we are on the conservative side in adopting the estimate $\mathcal{M}_{2 \mathrm{~B}}=(0.039 \sim 0.044) \mathrm{fm}$. Since the leading single-particle term is independent of $\Lambda$, the total amplitude $\mathcal{M}=\mathcal{M}_{1 \mathrm{~B}}+\mathcal{M}_{2 \mathrm{~B}}$ is $\Lambda$-independent to the same degree as $\mathcal{M}_{2 \mathrm{~B}}$. The $\Lambda$-independence of the physical quantity $\mathcal{M}$, which is in conformity with the tenet of EFT, is a crucial feature of the result in our present study. The relative strength of the two-body contribution as compared with the one-body contribution is

$$
\delta_{2 \mathrm{~B}} \equiv \frac{\mathcal{M}_{2 \mathrm{~B}}}{\mathcal{M}_{1 \mathrm{~B}}}=(0.86 \pm 0.05) \% .
$$

We remark that the central value of $\delta_{2 \mathrm{~B}}$ here is considerably smaller than the corresponding value, $\delta_{2 \mathrm{~B}}=4 \%$, in PKMR98. Furthermore, the uncertainty of $\pm 0.05 \%$ in Eq.(28) is drastically smaller than the corresponding figure, $\pm 4 \%$, in PKMR98.

We now turn to the threshold $S$ factor, $S_{p p}(0)$. Adopting the value $G_{V}=(1.14939 \pm 0.00065) \times 10^{-5} \mathrm{GeV}^{-2}$ [36], we obtain

$$
\begin{aligned}
S_{p p}(0) & =3.94 \times\left(\frac{1+\delta_{2 B}}{1.01}\right)^{2}\left(\frac{g_{A}}{1.2670}\right)^{2}\left(\frac{\Lambda_{p p}^{2}}{6.91}\right)^{2} \\
& =3.94 \times(1 \pm 0.0015 \pm 0.0010 \pm \varepsilon)
\end{aligned}
$$

in units of $10^{-25} \mathrm{MeV}-\mathrm{b}$. Here the first error is due to uncertainties in the input parameters in the one-body part, while the second error represents the uncertainties in the two-body part; $\varepsilon(\approx 0.001)$ denotes possible uncertainties due to higher chiral order contributions (see below). To make a formally rigorous assessment of $\varepsilon$, we must evaluate loop corrections and higher-order counter terms. Although an $\mathrm{N}^{4} \mathrm{LO}$ calculation would not involve any new unknown parameters, it is a non-trivial task. Furthermore, loop corrections necessitate a more elaborate regularization scheme since the naive cutoff regularization used here violates chiral symmetry at loop orders. (This difficulty, however, is not insurmountable.) These formal problems set aside, it seems reasonable to assess $\varepsilon$ as follows. We first recall that both tritium $\beta$-decay and solar $p p$ fusion are dominated by the one-body GT matrix elements, the evaluation of which is extremely well controlled from the SNPA as well as EFT points of view. Therefore, the precision of our calculation is governed by the reliability of estimation of small corrections to the

TABLE II. The strength $\hat{d}^{R}$ of the contact term and the two-body GT matrix element, $\mathcal{M}_{2 \mathrm{~B}}$, for the $p p$ process calculated for representative values of $\Lambda$.

\begin{tabular}{|c|c|l|}
\hline \hline$\Lambda(\mathrm{MeV})$ & $\hat{d}^{R}$ & $\mathcal{M}_{2 \mathrm{~B}}(\mathrm{fm})$ \\
\hline 500 & $1.00 \pm 0.07$ & $0.076-0.035 \hat{d}^{R} \simeq 0.041 \pm 0.002$ \\
\hline 600 & $1.78 \pm 0.08$ & $0.097-0.031 \hat{d}^{R} \simeq 0.042 \pm 0.002$ \\
\hline 800 & $3.90 \pm 0.10$ & $0.129-0.022 \hat{d}^{R} \simeq 0.042 \pm 0.002$ \\
\hline \hline
\end{tabular}

dominant one-body GT contribution. Now, we have seen that the results of the present $\mathrm{N}^{3} \mathrm{LO}$ calculation nicely fit into the picture expected from the general premise of EFT: (i) the $\mathrm{N}^{3} \mathrm{LO}$ contributions are indeed much smaller than the leading order term; (ii) the physical transition amplitude $\mathcal{M}$ does not depend on the cutoff parameter. Although these features do not constitute a formal proof of the convergence of the chiral expansion used here, it is extremely unlikely that higher order contributions be so large as to completely upset the physically reasonable behavior observed in the $\mathrm{N}^{3} \mathrm{LO}$ calculation. It should therefore be safe to assign to $\varepsilon$ an uncertainty comparable to the error estimate for the two-body part in Eq.(29); viz., $\varepsilon \approx 0.1 \%$. In this connection we remark that an axial three-body MEC contribution to the ${ }^{3} \mathrm{H}$ GT matrix element was calculated explicitly in SNPA [18 and found to be negligible relative to the leading two-body mechanisms. This feature is consistent with the above argument since, in the context of EFT, the three-body MEC represents a higher-order effect subsumed in " $\varepsilon$ " in Eq.(29).

Apart from the notable numerical differences between the present work and PKMR98, it is worth noting that short-range physics is much better controlled in MEEFT. In the conventional treatment of MEC, one derives the coordinate space representation of a MEC operator by applying ordinary Fourier transformation (with no restriction on the range of the momentum variable) to the amplitude obtained in momentum space; this corresponds to setting $\Lambda=\infty$ in Eq.(21). In PKMR98, where this familiar method is adopted, the $\hat{d}^{R}$ term appears in the zero-range form, $\hat{d}^{R} \delta(r)$. PKMR98 chose to introduce short-range repulsive correlation with hardcore radius $r_{C}$ and eliminate the $\hat{d}^{R} \delta(r)$ term by hand. The remaining finite-range terms were evaluated as functions of $r_{C} . \mathcal{M}_{2 \mathrm{~B}}$ calculated this way exhibited substantial $r_{C}$-dependence, indicating that short-range physics was not well controlled. Inclusion of the $\hat{d}^{R}$ term, with its strength renormalized as described here, properly takes into account the short-range physics inherited from the integrated out degrees of freedom above the cutoff, thereby drastically reducing the undesirable (or unphysical) sensitivity to short-distance physics.

\section{THE hep PROCESS}

In the notation of MSVKRB, the GT-amplitudes are given in terms of the reduced matrix elements, $\bar{L}_{1}(q ; A)$ and $\bar{E}_{1}(q ; A)$. Since these matrix elements are related to each other as $\bar{E}_{1}(q ; A) \simeq \sqrt{2} \bar{L}_{1}(q ; A)$, with the exact equality holding at $q=0$, we consider here only one of them, $\bar{L}_{1}(q ; A)$. For the three exemplary values of $\Lambda$, Table III] gives the corresponding values of $\bar{L}_{1}(q ; A)$ at $q \equiv|\boldsymbol{q}|=19.2 \mathrm{MeV}$ and zero c.m. energy; for conve- 
nience, the values of $\hat{d}^{R}$ in Eq.(23) are also listed. We see from the table that the variation of the two-body GT amplitude (row labelled "2B-total") is only $\sim 10 \%$ for the range of $\Lambda$ under study. Note that the $\Lambda$-dependence in the total GT amplitude is made more pronounced by the drastic cancellation between the one-body and two-body terms, but this amplified $\Lambda$-dependence still lies within acceptable levels.

TABLE III. Values of $\hat{d}^{R}$ and $\bar{L}_{1}(q ; A)\left(\right.$ in $\left.\mathrm{fm}^{3 / 2}\right)$ for the hep process calculated as functions of the cutoff $\Lambda$. The individual contributions from the one-body (1B) and two-body (2B) operators are also listed.

\begin{tabular}{c|rrr}
\hline \hline$\Lambda(\mathrm{MeV})$ & 500 & 600 & 800 \\
\hline$\hat{d}^{R}$ & $1.00 \pm 0.07$ & $1.78 \pm 0.08$ & $3.90 \pm 0.10$ \\
\hline $\bar{L}_{1}(q ; A)$ & -0.032 & -0.029 & -0.022 \\
\hline $1 \mathrm{~B}$ & -0.081 & -0.081 & -0.081 \\
\hline 2B (without $\left.\hat{d}^{R}\right)$ & 0.093 & 0.122 & 0.166 \\
2B $\left(\propto \hat{d}^{R}\right)$ & -0.044 & -0.070 & -0.107 \\
\hline 2B-total & 0.049 & 0.052 & 0.059 \\
\hline \hline
\end{tabular}

TABLE IV. Contributions to $S_{h e p}(0)$ (in $10^{-20} \mathrm{keV}-\mathrm{b}$ ) from individual initial channels calculated as functions of $\Lambda$. The last column gives the results obtained in MSVKRB.

\begin{tabular}{c|ccc|c}
\hline \hline$\Lambda(\mathrm{MeV})$ & 500 & 600 & 800 & MSVKRB \\
\hline${ }^{1} S_{0}$ & 0.02 & 0.02 & 0.02 & 0.02 \\
${ }^{3} S_{1}$ & 7.00 & 6.37 & 4.30 & 6.38 \\
${ }^{3} P_{0}$ & 0.67 & 0.66 & 0.66 & 0.82 \\
${ }^{1} P_{1}$ & 0.85 & 0.88 & 0.91 & 1.00 \\
${ }^{3} P_{1}$ & 0.34 & 0.34 & 0.34 & 0.30 \\
${ }^{3} P_{2}$ & 1.06 & 1.06 & 1.06 & 0.97 \\
\hline Total & 9.95 & 9.37 & 7.32 & 9.64 \\
\hline \hline
\end{tabular}

Table IV shows the contribution to the $S$-factor, at zero c.m. energy, from each initial channel. For comparison we also give the results of MSVKRB for the AV18/UIX interaction. It is noteworthy that for all the channels other than ${ }^{3} S_{1}$, the $\Lambda$-dependence is very small ( $\lesssim 2 \%$ ). The ${ }^{3} S_{1}$ channel is the most sensitive to shortdistance physics because the extraordinary suppression of the one-body GT contribution makes more pronounced the chiral-non-protected nature of the GT transition. In fact, the sensitivity of the ${ }^{3} S_{1}$ channel to short-distance physics would be larger if the contribution of the $A^{0}$ term, which is rather sizable here despite its generic $1 / m$ suppression, were omitted. It is therefore reassuring that the chiral-filter mechanism allows reliable estimation of the $A^{0}$ term in this channel as well (besides the $P$-wave channels), see [18].

Summarizing the results given in Table IV, we arrive at a prediction for the hep $S$-factor 17 :

$$
S_{h e p}(0)=(8.6 \pm 1.3) \times 10^{-20} \mathrm{keV}-\mathrm{b},
$$

where the "error" spans the range of the $\Lambda$-dependence for $\Lambda=500-800 \mathrm{MeV}$. This result should be compared to that obtained by MSVKRB [18], $S_{h e p}(0)=9.64 \times$ $10^{-20} \mathrm{keV}-\mathrm{b}$

The latest analysis of the Super-Kamiokande data 37] gives an upper limit of the solar hep neutrino flux, $\Phi(h e p)^{\mathrm{SK}}<40 \times 10^{3} \mathrm{~cm}^{-2} \mathrm{~s}^{-1}$. The standard solar model [38 using the hep $S$-factor of MSVKRB [18 predicts $\Phi(h e p)^{\mathrm{SSM}}=9.4 \times 10^{3} \mathrm{~cm}^{-2} \mathrm{~s}^{-1}$. The use of the central value of our estimate, Eq.(30), of the hep $S$-factor would slightly lower $\Phi(h e p)^{\mathrm{SSM}}$ but with the upper limit compatible with $\Phi(h e p)^{\mathrm{SSM}}$ in Ref. [38]. A significantly improved estimate of $S_{h e p}(0)$ in Eq.(30) is expected to be useful for further discussion of the solar hep problem.

To reduce the uncertainty in Eq. (30), we need to reduce the $\Lambda$-dependence in the two-body GT term. According to a general tenet of EFT, the cutoff dependence should diminish as higher order terms get included. In fact, the somewhat rapid variation seen in $\hat{d}^{R}$ (Table III) and in the ${ }^{3} S_{1}$ contribution to $S_{h e p}(0)$ (Table IV) as $\Lambda$ approaches $800 \mathrm{MeV}$ may be an indication that there is need for the explicit presence of the vector-mesons ( $\rho$ and $\omega)$ with mass $m_{V} \lesssim \Lambda$. This possible insufficiency could be remedied to a certain extent by going to higher orders. A preliminary study [39] indicates that it is indeed possible to reduce the $\Lambda$-dependence significantly by including $\mathrm{N}^{4} \mathrm{LO}$ corrections. We expect that the higher order correction would make the result for $\Lambda=800 \mathrm{MeV}$ closer to those for $\Lambda=500,600 \mathrm{MeV}$, bringing the MEEFT results closer to what was obtained in MSVKRB. This possibility is taken into account in the error estimate given in Eq.(30).

\section{DISCUSSION}

It is worth emphasizing that the above MEEFT prediction for $\delta_{2 \mathrm{~B}}$ for the $p p$ process is in line with the latest SNPA results obtained in Ref. [25] (and mentioned earlier). There too, the short range behavior of the axial MEC was constrained by reproducing $\Gamma_{\beta}^{t}$. The inherent model dependence of such a procedure within the SNPA context was shown to be very weak simply because at small inter-particle separations, where MEC contributions are largest, the pair wave functions in different

\footnotetext{
${ }^{11}$ See below for a possible caveat on the given error estimate. ${ }^{12}$ The earlier studies 16.17 were based on less accurate variational wave functions than used here and in MSVKRB; furthermore they did not include P-wave capture contributions, which account for $\approx 40 \%$ of the total $S$-factor.
} 
nuclei are similar in shape and differ only by a scale factor [40]. As a consequence, the ratios of GT and $p p$ capture matrix elements of different two-body current terms are nearly the same, and therefore a knowledge of their sum in the GT matrix element is sufficient to predict their sum in the $p p$-capture matrix element [25].

In order to better understand how the present scheme works, it is helpful to compare the hep reaction with the radiative $n p$-capture. The polarization observables in $\vec{n}+\vec{p} \rightarrow d+\gamma$ are known to be sensitive to the isoscalar M1 matrix element, $M 1 S$, and this amplitude has been extensively studied in EFT [22,41]. The similar features of the hep GT amplitude and $M 1 S$ are: (i) the leading one-body contribution is suppressed by the symmetries of the wave functions; (ii) there is no soft-pion exchange contribution; (iii) nonetheless, short-range physics can be reliably subsumed into a single contact term. In the $\vec{n} \vec{p}$ case the strength of this term can be determined from the deuteron magnetic moment (for a given value of $\Lambda$ ). The calculation in Ref. [22] demonstrates that the $\Lambda$ dependence in the contact term and that of the remaining terms compensate each other so that the total $M 1 S$ is stable against changes in $\Lambda$. This suggests that, if we go to higher orders, the coefficient of the contact term in question will be modified, with part of its strength shifted to higher order terms; however, the total physical amplitude will remain essentially unchanged. These features are quite similar to what we have found here for the hep GT amplitude.

Evaluating the matrix element of the leading-order one-body operator in EFT with the use of realistic nuclear wave functions is analogous to fixing parameters in an EFT Lagrangian (at a given order) using empirical inputs [42]; the realistic wave functions in SNPA can be regarded as a theoretical input that fits certain sets of observables. In the present MEEFT scheme, we take the view that the same realistic wave functions also provide a framework for reliably calculating (finite-range) manybody corrections to the leading-order one-body matrix element. The short-ranged part inherited from the integrated out degrees of freedom is regulated by the $\hat{d}^{R}$ term. This way of handling "short-range correlation" is analogous to Bogner et al.'s derivation [5] of " $V_{\text {low }-k}$ " based on renormalization-group theory. While our approach here is, in certain cases, not in strict accordance with the systematic power-counting scheme of EFT proper, nevertheless the severity of this potential shortcoming should vary from one case to another (see discussion in Ref. [43|). For the $p p$ and hep amplitudes under consideration, the degree of $\Lambda$-dependence exhibited by the numerical results does suggest that deviations from rigorous powercounting cannot be too significative. Indeed, this type of "resilience" may also explain why the SNPA calculation in Ref. 18 gives a result very similar to the present one. It is true that the two-body terms in MSVKRB are not entirely in conformity with the chiral counting scheme we are using here; some terms corresponding to chiral orders higher than $\mathrm{N}^{3} \mathrm{LO}$ are included, while some other terms which are $\mathrm{N}^{3} \mathrm{LO}$ in EFT are missing (see Appendix B.3). Most importantly the $\hat{d}^{R}$-term - that plays a crucial role here - is omitted in MKSVRB although heavy-meson exchange graphs may account for some part of it. This formal problem, however, seems to be largely overcome by the fact that also in MSVKRB a parameter (the axial $N \Delta$ coupling strength) is adjusted to reproduce $\Gamma_{\beta}^{t}$.

Not unrelated to the above issue of power-counting is the question of consistency of embedding "realistic" wave functions obtained from "realistic" potentials that are fitted accurately to experiments into an EFT framework with the currents obtained to a given order of chiral perturbation theory. It is a well-known fact that potentials that fit experiments are not necessarily unique. The non-uniqueness resides however in the short-range part of the potential, with the long-range part primarily governed by the pion exchange. Let us suppose that one can calculate potentials to a very high order in a consistent expansion (that is, consistent with symmetries etc.). The structure of the potential would depend on various aspects of the calculation. For instance, although they all may fit equally well various experimental data such as e.g., nucleon-nucleon scattering, different regularizations would lead to different potentials, the difference residing mainly in the short-range part. One might worry that this non-uniqueness would upset the basic premise of an EFT, rendering the predictions untrustworthy.

Another intricate issue, which is also connected to short-range physics, is the off-shell ambiguity. This problem should be absent in a formally consistent EFT. In MEEFT, however, we insert the current operators derived from irreducible diagrams up to a given chiral order between phenomenological (albeit realistic) wave functions. Since the inserted current involves off-shell particles, there can in principle be terms other than those that have been included in our approach. While those additional terms that may be required to eliminate the off-shell dependence are expected to be of higher order than $\mathrm{N}^{3} \mathrm{LO}$, this issue warrants a further examination.

To answer the above question with full rigor, much more work is needed. However, partial and yet reasonably satsifactory answers can be obtained from this work. For chiral-filter protected processes, we have presented a clear argument that the above-mentioned ambiguity does not matter at the level of accuracy in question. The results listed in Table IV for the $P$-wave capture (to which the chiral-protected time component of the axial current contributes) demonstrate this point. The question of short-distance ambiguity arises only for chiralunprotected processes like the GT transition. As already explained, however, the $\hat{d}^{R}$ regularization essentially removes this ambiguity. The point is that the physics of the degrees of freedom above the cutoff scale $\Lambda$ gets lodged 
in the short-range $\hat{d}^{R}$ term. In fixing this term as a function of $\Lambda$ via the experimental value of $\Gamma_{\beta}^{t}$, one is essentially incorporating the short-range correlations that render low-energy physics insensitive to short-distance physics.

As for the off-shell problem, we note that for processes involving a long-wavelength external current - such as the solar $p p$ and hep reactions - the off-shell ambiguity should be small, so long as one uses high-quality phenomenological wave functions that accurately describe processes without the external current. The wave functions used here describe with high accuracy a rich ensemble of data available for the systems in question; they describe very well the three-nucleon scattering states, and furthermore, the $n^{3} \mathrm{He}$ elastic scattering cross section as well as the coherent scattering length calculated with these wave functions are in excellent agreement with the experiments. What is involved here seems to be a generic feature. A similar stabilizing mechanism is at work when Bogner et al. [5] arrive at a unique effective force $V_{l o w-k}$ by integrating out the high-energy/momentum components contained in various "realistic" potentials. Nuclear physics calculations done with this effective force 44] have much in common with the MEEFT calculation described here. Furthermore, we remark that different offshell properties reflect different choices of the field variables and that, for each choice, the LECs need to be readjusted. It is in principle possible to choose the field variables in such a manner that off-shell contributions become highly suppressed. We are essentially adopting this particular choice by using the forms of the transition operators described above and adjusting the corresponding LEC, $\hat{d}^{R}$, to reproduce $\Gamma_{\beta}^{t}$.

A possible approach that is formally consistent with systematic power counting is the pionless EFT based on the power divergence subtraction (PDS) scheme 45 (for a recent review, see Ref. [46]), which has been applied to the $p p$ fusion [47]. Due to the fact that this scheme also involves one unknown low-energy constant, PDS has not so far led to a definite prediction on the $p p$ fusion rate. The problem is that this approach cannot be readily extended to systems with $A \geq 3$, in particular to electroweak transition amplitudes in these systems. What is lacking presently is a method to correlate in a unified framework the observables in different nuclei (different mass numbers). This limitation keeps one from exploiting the experimental data available for the $A \geq 3$ nuclei to fix unknown LEC. Apart from the basic problem of organizing chiral expansion for complex nuclei from "first-principles", a plethora of parameters involved would present a major obstacle. (For recent efforts in this approach, see Ref. [46, 48] and references given therein.) This difficulty is expected to be particularly pronounced for the hep reaction.

There has been a series of intensive studies by the Jülich Group to extend EFT calculations in the Weinberg scheme to systems with three or more nucleons 49 . The relationship between this approach and the phenomenological potential approach has been examined in great detail. This line of study, however, has been so far limited to nuclear observables that do not involve the electroweak currents. An extension of the formalism developed in Ref. 49] to electroweak transitions should be extremely useful.

\section{ACKNOWLEDGEMENT}

TSP and KK thank S. Ando and F. Myhrer for discussions. MR is grateful for clarifying discussions with Gerry Brown on the notion of "double decimation" in nuclear structure theory. The work of TSP and KK is supported in part by the U.S. National Science Foundation, Grant No. PHY-9900756 and No. INT-9730847. The work of RS is supported by DOE contract No. DE-AC0584ER40150, under which the Southeastern Universities Research Association (SURA) operates the Thomas Jefferson National Accelerator Facility. The work of DPM is supported in part by KOSEF Grant 1999-2-111-005-5 and KSF Grant 2000-015-DP0072. MR acknowledges the hospitality of the Physics Departments of the Seoul National University and Yonsei University, where his work was partially supported by Brain Korea 21 in 2001. TSP and KK would like to thank Korea Institute for Advanced Study for the hospitality while part of this paper was being written. Some of the calculations were made possible by grants of computing time from the National Energy Research Supercomputer Center in Livermore.

\section{APPENDIX A: GAMOW-TELLER OPERATORS}

The aim of this and subsequent Appendices is to provide some technical details that have been left out in the main text. The readers who are not interested in the details of our calculation can safely skip these Appendices without missing the essential points of our results.

We decompose the axial current into $n_{B}$-body operators as

$$
\begin{aligned}
A^{\mu, a}= & A_{1 \mathrm{~B}}^{\mu, a}+A_{2 \mathrm{~B}}^{\mu, a}+A_{3 \mathrm{~B}}^{\mu, a}+\cdots \\
= & \sum_{l} A_{l}^{\mu, a}+\sum_{l<m} A_{l m}^{\mu, a}+\sum_{l<m<n} A_{l m n}^{\mu, a} \\
& +\cdots,
\end{aligned}
$$

where $(l, m, n)$ are particle indices. The one-body operator can be read from

$$
\begin{aligned}
& \left\langle N\left(p^{\prime}\right)\left|A^{\mu, a}(x=0)\right| N(p)\right\rangle \\
& =-\bar{u}\left(p^{\prime}\right)\left[G_{A}\left(k^{2}\right) \gamma^{\mu} \gamma_{5}-\frac{G_{P}\left(k^{2}\right)}{2 m_{N}} k^{\mu} \gamma_{5}\right] \frac{\tau^{a}}{2} u(p),
\end{aligned}
$$


where $u(p)$ is a four-component Dirac spinor of momentum $p$, and $k^{\mu}=\left(p-p^{\prime}\right)^{\mu}$ is the momentum carried by the lepton pair. $G_{A}\left(k^{2}\right)$ and $G_{P}\left(k^{2}\right)$ are the axial and induced pseudoscalar form factors, respectively. Note that $k^{\mu}=\mathcal{O}\left(Q^{2} / m_{N}\right)$, while $\boldsymbol{p}=\mathcal{O}(Q)$ and $\boldsymbol{p}^{\prime}=\mathcal{O}(Q)$. Thus the contribution from $G_{P}$ term is of $\mathcal{O}\left(Q^{4}\right)$. In getting the non-relativistic operators from the above relativistic form factors, we should also take into account the wave function normalization. The resulting one-body operator up to $\mathcal{O}\left(Q^{3}\right)$ then reads

$$
\begin{aligned}
\boldsymbol{A}_{l}^{a}= & -\frac{\tau_{l}^{a}}{2} g_{A} \mathrm{e}^{-i \boldsymbol{r}_{l} \cdot \boldsymbol{q}}\left[\boldsymbol{\sigma}_{l}+\frac{\overline{\boldsymbol{p}}_{l} \boldsymbol{\sigma}_{l} \cdot \overline{\boldsymbol{p}}_{l}-\boldsymbol{\sigma}_{l} \overline{\boldsymbol{p}}_{l}^{2}}{2 m_{N}^{2}}\right. \\
& \left.+\frac{i \boldsymbol{q} \times \boldsymbol{p}_{l}}{4 m_{N}^{2}}+\mathcal{O}\left(\frac{Q^{4}}{m_{N}^{4}}\right)\right] .
\end{aligned}
$$

This expression has appeared in eq.(16).

In the following subsections, we derive all the two-body GT operators up to $\mathrm{N}^{4} \mathrm{LO}$ and leading three-body GT operators .

\section{Two-body GT}

There are no two-body GT diagrams that involve only $\nu_{i}=0$ vertices, because the $\mathcal{A}^{i, a} \pi N N$ vertex is kinematically suppressed, and there is no four-fermion contact contribution at LO $\left(\nu_{i}=0\right)$. As a consequence, the twobody GT operator starts at $\nu=3$. The two-body GT operator at threshold $\left(q^{\mu} \rightarrow 0\right)$ was given up to $\mathrm{N}^{3} \mathrm{LO}$ in Ref. [50]. We extend here that analysis to include all contributions up to $\mathrm{N}^{4} \mathrm{LO}$. To this end, it is useful to decompose $\boldsymbol{A}_{l m}^{a}$ as

$$
\boldsymbol{A}_{l m}^{a}=\boldsymbol{A}_{l m}^{a}(1 \pi)+\boldsymbol{A}_{l m}^{a}(2 \pi),
$$

where $\boldsymbol{A}_{l m}^{a}(1 \pi)$ represents the contributions of the onepion pole part and $\boldsymbol{A}_{l m}^{a}(2 \pi)$ stands for the remaining short-ranged part. Generic diagrams for $\boldsymbol{A}_{l m}^{a}(1 \pi)$ and $\boldsymbol{A}_{l m}^{a}(2 \pi)$ are shown in Fig. 1.

We now list all the two-body GT operators belonging to $\nu=3$ and $\nu=4$.

- $\nu=3$ :

This contribution comes from tree graphs (one-pionexchange and contact) with a $\nu_{i}=1$ vertex. The resulting GT operators, given in Ref. [50], are of the form

$$
\begin{aligned}
\boldsymbol{A}_{12}^{a: \nu 3}(1 \pi) & =\frac{g_{A}}{2 m_{N} f_{\pi}^{2}}\left[\frac{i}{2}\left(\vec{\tau}_{1} \times \vec{\tau}_{2}\right)^{a} \overline{\boldsymbol{p}}_{1}+2 \hat{c}_{3} \tau_{2}^{a} \boldsymbol{k}_{2}\right. \\
& +\left(\hat{c}_{4}+\frac{1}{4}\right)\left(\vec{\tau}_{1} \times \vec{\tau}_{2}\right)^{a} \boldsymbol{\sigma}_{1} \times \boldsymbol{k}_{2} \\
& \left.+\frac{1+c_{6}}{4}\left(\vec{\tau}_{1} \times \vec{\tau}_{2}\right)^{a} \boldsymbol{\sigma}_{1} \times \boldsymbol{q}\right] \frac{\boldsymbol{\sigma}_{2} \cdot \boldsymbol{k}_{2}}{\boldsymbol{k}_{2}^{2}+m_{\pi}^{2}} \\
& +(1 \leftrightarrow 2), \\
\boldsymbol{A}_{12}^{a: \nu 3}(2 \pi) & =\frac{g_{A}}{m_{N} f_{\pi}^{2}}\left[\hat{d}_{1}\left(\tau_{1}^{a} \boldsymbol{\sigma}_{1}+\tau_{2}^{a} \boldsymbol{\sigma}_{2}\right)\right.
\end{aligned}
$$

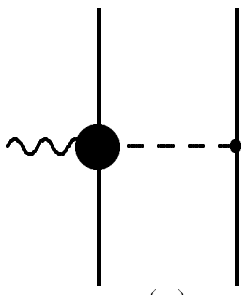

(a)

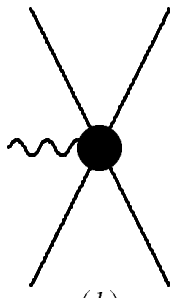

(b)
FIG. 1. A one-pion pole diagram, (a), responsible for $\boldsymbol{A}_{l m}^{a}(1 \pi)$, and a short-range contribution diagram, $(b)$, responsible for $\boldsymbol{A}_{l m}^{a}(2 \pi)$. The solid circles include counter-term insertions and (one-particle irreducible) loop corrections. The wiggly line stands for the external field (current) and the dashed line for the pion. One-loop corrections of the relevant orders for the pion propagator and the $\pi N N$ vertex need to be included.

$$
\left.+\hat{d}_{2}\left(\vec{\tau}_{1} \times \vec{\tau}_{2}\right)^{a} \sigma_{1} \times \boldsymbol{\sigma}_{2}\right] .
$$

Although there are two unknown parameters, $\hat{d}_{1}$ and $\hat{d}_{2}$, it turns out that the Fermi-Dirac statistics effectively reduces the number of unknowns to one. We will come back to this important point later.

- $\nu=4$ :

Tree graphs with $\sum_{i} \nu_{i}=2$ and one-loop graphs with $\sum_{i} \nu_{i}=0$ enter at this order. Since there is no $\pi N N$ vertex with $\nu_{i}=1$, a $\nu=4$ tree graph should have either $\boldsymbol{A}(N N)^{2}$ or $\boldsymbol{A} \pi N N$ vertex with $\nu_{i}=2$. We can, however, exclude either possibility. The absence of $\boldsymbol{A} \pi N N$ vertex at $\nu_{i}=2$ can be ascertained by consulting a complete list of terms that appear in the $\mathrm{N}^{2} \mathrm{LO}$ Lagrangian given in Ref. [51]. As for the $\nu_{i}=2 \boldsymbol{A}(N N)^{2}$ vertex for the two-nucleon sector, a complete list is not available yet. We therefore resort to parity selection rules. Our vertex must have one $\Delta_{\mu}$ and one $D_{\mu}$ involving four nucleon fields. These operators should not be contracted with the four-velocity $v^{\mu}$, for otherwise the actual chiral index would acquire an extra power of $Q$. We can easily show, however, that it is impossible to construct a parity-even Lorentz scalar with one $\Delta_{\mu}$, one $D_{\mu}$ and arbitrary numbers of $S^{\mu}$ and $\epsilon^{\mu \nu \alpha \beta}$. Introducing an operator of the $\partial_{\mu} A_{\nu}-\partial_{\nu} A_{\mu}$ type instead of $\Delta_{\mu}$ and $D_{\mu}$ does not help either. These observations lead us to conclude that no divergences occur in the relevant loops and, more importantly, that no new parameters appear at $\nu=4$.

The one-pion-exchange contribution can be read off from 1-loop corrections to the $\Gamma_{\mathcal{A} \pi}^{\mu, a b}$ vertex; the relevant diagrams are shown in Fig. 2. We note that only the first five diagrams $(a)-(e)$ contribute to $\boldsymbol{A}$. Using the expressions given in Ref. [15], where all the one-loop diagrams have been calculated, we find

$$
\boldsymbol{A}_{12}^{a: \nu 4}(1 \pi)=-\frac{g_{A}^{3}}{32 \pi f_{\pi}^{4}}
$$




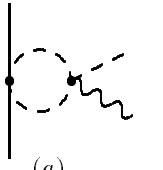

(a)
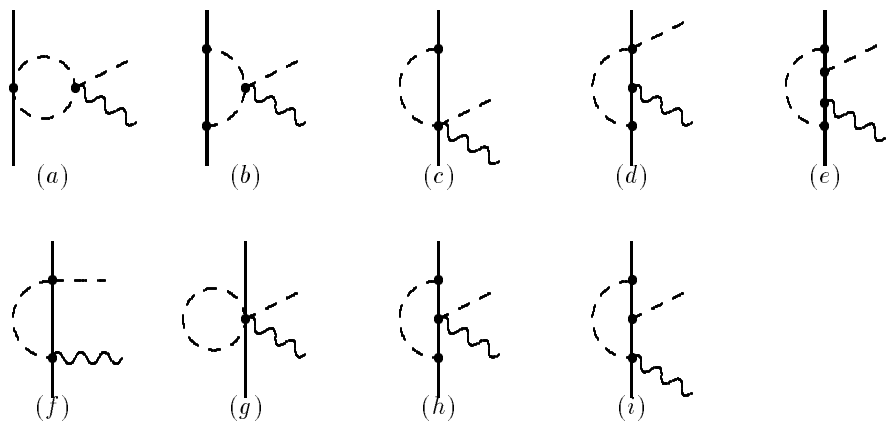

FIG. 2. One loop diagrams that contribute to the $\mathcal{A}^{\mu} \pi N N$ vertex. Only the first five diagrams $(a)-(e)$ contribute to $\boldsymbol{A}$.

$$
\begin{aligned}
& \otimes\left[\left(\vec{\tau}_{1} \times \vec{\tau}_{2}\right)^{a}\left(\boldsymbol{\sigma}_{1} \times\left(\boldsymbol{k}_{2}+\boldsymbol{q}\right) D_{1}\left(k_{1}\right)+\frac{g_{A}^{2}}{8} \boldsymbol{\sigma}_{1} \times \boldsymbol{k}_{2} m_{\pi}\right)\right. \\
& -\tau_{2}^{a}\left(\left(\boldsymbol{q}+3 \boldsymbol{k}_{2}\right)\left[D_{1}\left(k_{1}\right)+\frac{1}{3} \boldsymbol{k}_{1}^{2} D_{2}\left(k_{1}\right)\right]+\frac{9 g_{A}^{2}}{8} \boldsymbol{k}_{2} m_{\pi}\right) \\
& ] \frac{\boldsymbol{\sigma}_{2} \cdot \boldsymbol{k}_{2}}{m_{\pi}^{2}+\boldsymbol{k}_{2}^{2}}+(1 \leftrightarrow 2),
\end{aligned}
$$

where $k_{l}=\left|\boldsymbol{k}_{l}\right|(l=1,2)$, and $D_{i}(k)$ 's are defined as

$$
\begin{aligned}
D_{1}(k) & =\int_{0}^{1} d z M_{z k} \\
D_{2}(k) & =\int_{0}^{1} d z \frac{z \bar{z}}{M_{z k}} \\
D_{3}(k) & =\int_{0}^{1} d z\left(-\frac{z \bar{z} \boldsymbol{k}^{2}}{M_{z k}}-5 M_{z k}\right) \\
& =4 \int_{0}^{1} d z\left(-\frac{3}{2} M_{z k}+\frac{m_{\pi}^{2}}{4 M_{z k}}\right), \\
D_{4}(k) & =\int_{0}^{1} d z\left(-\frac{(z \bar{z})^{2} \boldsymbol{k}^{2}}{M_{z k}^{3}}+7 \frac{z \bar{z}}{M_{z k}}-\frac{1}{M_{z k}}\right) \\
& =4 \int_{0}^{1} d z\left(\frac{z \bar{z}}{2 M_{z k}}+\frac{z \bar{z} m_{\pi}^{2}}{4 M_{z k}^{3}}-\left(\frac{1}{4}-z \bar{z}\right) \frac{1}{M_{z k}}\right) \\
D_{5}(k) & =\int_{0}^{1} d z \frac{1}{M_{z k}}, \\
D_{6}(k) & =\int_{0}^{1} d z\left(\frac{1}{4}-z \bar{z}\right) \frac{1}{M_{z k}} .
\end{aligned}
$$

Here $k \equiv|\boldsymbol{k}|, \bar{z} \equiv 1-z$, and $M_{z k} \equiv \sqrt{m_{\pi}^{2}+z \bar{z} \boldsymbol{k}^{2}}$.

The integrations over $z$ can be done analytically, resulting in

$$
\begin{aligned}
& D_{1}(k)=\frac{m_{\pi}}{2}+\frac{4 m_{\pi}^{2}+k^{2}}{4 k} \Theta_{k}, \\
& D_{2}(k)=\frac{m_{\pi}}{2 k^{2}}-\frac{4 m_{\pi}^{2}-k^{2}}{4 k^{3}} \Theta_{k}, \\
& D_{3}(k)=-3 m_{\pi}-\frac{8 m_{\pi}^{2}+3 k^{2}}{2 k} \Theta_{k}, \\
& D_{4}(k)=\frac{1}{2 k^{3}}\left[\frac{2 m_{\pi} k\left(8 m_{\pi}^{2}+3 k^{2}\right)}{4 m_{\pi}^{2}+k^{2}}-\left(8 m_{\pi}^{2}+k^{2}\right) \Theta_{k}\right],
\end{aligned}
$$

$$
\begin{aligned}
& D_{5}(k)=\frac{2}{k} \Theta_{k}, \\
& D_{6}(k)=-\frac{m_{\pi} k}{2 k^{3}}+\frac{4 m_{\pi}^{2}+k^{2}}{4 k^{3}} \Theta_{k}
\end{aligned}
$$

where $k \equiv|\boldsymbol{k}|$ and

$$
\Theta_{k} \equiv \tan ^{-1} \frac{k}{2 m_{\pi}}
$$

with $-\frac{\pi}{2}<\Theta_{k}<\frac{\pi}{2}$.

Note that the one-pion-pole contributions can be absorbed into $\boldsymbol{A}_{l m}^{a: \nu 3}(1 \pi)$ (given in Eq.(A.5)) by renormalizing $\hat{c}_{3}$ and $\hat{c}_{4}$,

$$
\begin{aligned}
\hat{c}_{3} & \rightarrow \hat{c}_{3}^{R} \equiv \hat{c}_{3}+\frac{m_{N} g_{A}^{2}}{32 \pi f_{\pi}^{2}}\left[\tilde{D}_{1 \pi}+\frac{9 g_{A}^{2}}{8} m_{\pi}\right] \\
& \simeq \hat{c}_{3}+1.0334, \\
\hat{c}_{4} & \rightarrow \hat{c}_{4}^{R} \equiv \hat{c}_{4}-\frac{m_{N} g_{A}^{2}}{32 \pi f_{\pi}^{2}}\left[2 D_{1 \pi}+\frac{g_{A}^{2}}{8} m_{\pi}\right] \\
& \simeq \hat{c}_{4}-0.4821 .
\end{aligned}
$$

where

$$
\begin{aligned}
D_{1 \pi} & \left.\equiv D_{1}(k)\right|_{k^{2}=-m_{\pi}^{2}}=\frac{m_{\pi}}{4}\left[2+3 \tanh ^{-1} \frac{1}{2}\right] \\
\tilde{D}_{1 \pi} & \equiv 3 D_{1}(k)+\left.k^{2} D_{2}(k)\right|_{k^{2}=-m_{\pi}^{2}} \\
& =m_{\pi}\left[2+\tanh ^{-1} \frac{1}{2}\right] .
\end{aligned}
$$

For the two-pion contribution $\boldsymbol{A}_{l m}^{a: \nu 4}(2 \pi)$, the relevant one-loop graphs are shown in Fig. 3. Among the diagrams in the figure, only the first four graphs, $(a)-(d)$, can contribute to the GT operator; $(e)$ is identically zero due to isospin symmetry, and the remaining graphs, $(f)-(h)$, contribute only to $A^{0}$.

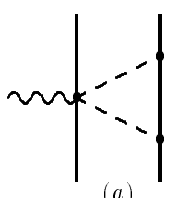

(a)
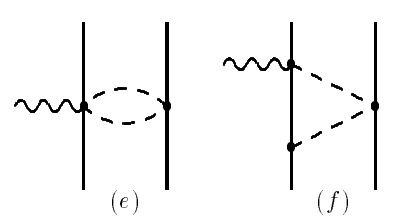

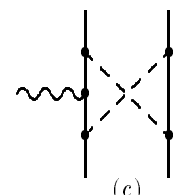

(c)

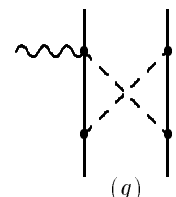

(g)
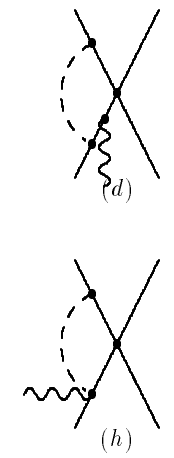

FIG. 3. One loop diagrams for the $\boldsymbol{A}_{l m}^{a: \nu 4}(2 \pi)$ : the first four diagrams $(a)-(d)$ contribute to the space part of the axial-vector (GT) and the remaining diagrams $(f)-(h)$ to the axial-charge current. The graph $(e)$ vanishes. 
As mentioned, the four diagrams $(a)-(d)$ are all ultraviolet-finite. The first three graphs give

$$
\begin{aligned}
\boldsymbol{A}_{12}^{a}(2 \pi: a) & =-\frac{g_{A}^{3}}{64 \pi f_{\pi}^{4}} \boldsymbol{\sigma}_{1} \tau_{1}^{a}\left[3 D_{1}\left(k_{2}\right)+\boldsymbol{k}_{2}^{2} D_{2}\left(k_{2}\right)\right] \\
& +(1 \leftrightarrow 2) \\
\boldsymbol{A}_{12}^{a}(2 \pi: b) & =-\frac{g_{A}^{3}}{128 \pi f_{\pi}^{4}} \tau_{2}^{a}\left[\boldsymbol{\sigma}_{1} D_{1}\left(k_{2}\right)\right. \\
& \left.-\left(2 \boldsymbol{k}_{2} \boldsymbol{\sigma}_{1} \cdot \boldsymbol{k}_{2}-\boldsymbol{\sigma}_{1} \boldsymbol{k}_{2}^{2}\right) D_{2}\left(k_{2}\right)\right] \\
& +(1 \leftrightarrow 2) \\
\boldsymbol{A}_{12}^{a}(2 \pi: c) & =-\frac{g_{A}^{5}}{1024 \pi f_{\pi}^{4}}\left(\tau_{1}^{a}+2 \tau_{2}^{a}\right)\left[\boldsymbol{\sigma}_{1} D_{3}\left(k_{2}\right)\right. \\
& +\left(2 \boldsymbol{k}_{2} \boldsymbol{\sigma}_{1} \cdot \boldsymbol{k}_{2}-\boldsymbol{\sigma}_{1} \boldsymbol{k}_{2}^{2}\right) D_{4}\left(k_{2}\right) \\
& \left.+\left(\boldsymbol{\sigma}_{2} \boldsymbol{k}_{2}^{2}-\boldsymbol{k}_{2} \boldsymbol{\sigma}_{2} \cdot \boldsymbol{k}_{2}\right) D_{5}\left(k_{2}\right)\right] \\
& +(1 \leftrightarrow 2)
\end{aligned}
$$

while the fourth diagram $(d)$ gives

$$
\begin{aligned}
\boldsymbol{A}_{12}^{a}(2 \pi: d)= & -\frac{g_{A}^{3} m_{\pi}}{64 \pi f_{\pi}^{2}} \sum_{A} \tau_{1}^{b} \sigma_{1}^{j}\left\{C_{A} \Gamma_{A} \Gamma_{A}, \tau_{1}^{a} \boldsymbol{\sigma}_{1}\right\} \tau_{1}^{b} \sigma_{1}^{j} \\
& +(1 \leftrightarrow 2) .
\end{aligned}
$$

The summation here is taken over all possible combinations of spin-isospin operators (with no derivatives) that figure in the nucleon-nucleon interactions. Using a generic expression

$$
\begin{aligned}
\sum_{A} C_{A} \Gamma_{A} \Gamma_{A} & =X_{1}+\boldsymbol{\sigma}_{1} \cdot \boldsymbol{\sigma}_{2} X_{\sigma}+\vec{\tau}_{1} \cdot \vec{\tau}_{2} X_{\tau} \\
& +\boldsymbol{\sigma}_{1} \cdot \boldsymbol{\sigma}_{2} \vec{\tau}_{1} \cdot \vec{\tau}_{2} X_{\sigma \tau}
\end{aligned}
$$

where $X_{1}, X_{\sigma}, X_{\tau}, X_{\sigma \tau}$ are constants that characterize the LO short-range nuclear forces, we can write

$$
\begin{aligned}
\boldsymbol{A}_{12}^{a}(2 \pi: d)=- & \frac{g_{A}^{3} m_{\pi}}{32 \pi f_{\pi}^{2}}\left[\left(-3 X_{1}+9 X_{\sigma \tau}\right)\left(\tau_{1}^{a} \boldsymbol{\sigma}_{1}+\tau_{2}^{a} \boldsymbol{\sigma}_{2}\right)\right. \\
& +\left(-2 X_{\sigma \tau}\right)\left(\vec{\tau}_{1} \times \vec{\tau}_{2}\right)^{a}\left(\boldsymbol{\sigma}_{1} \times \boldsymbol{\sigma}_{2}\right) \\
& \left.+\left(9 X_{\sigma}-3 X_{\tau}\right)\left(\tau_{1}^{a} \boldsymbol{\sigma}_{2}+\tau_{2}^{a} \boldsymbol{\sigma}_{1}\right)\right] .
\end{aligned}
$$

We demonstrate below that $X$ 's can all be absorbed into the parameters, $\hat{d}$ 's.

\section{Three-body GT}

The three-body GT operators up to $\mathrm{N}^{4} \mathrm{LO}$ come from the two diagrams given in Fig. 4. They contain only $\nu_{i}=0$ vertices, and their contributions read

$$
\begin{aligned}
\boldsymbol{A}_{123}^{a}= & -\sum_{\operatorname{cycle}(123)} \frac{g_{A}^{3}}{16 f_{\pi}^{4}} \\
& \otimes\left(2 \tau_{1}^{a} \vec{\tau}_{2} \cdot \vec{\tau}_{3}-\tau_{2}^{a} \vec{\tau}_{3} \cdot \vec{\tau}_{1}-\tau_{3}^{a} \vec{\tau}_{1} \cdot \vec{\tau}_{2}\right) \\
& \otimes\left(\boldsymbol{\sigma}_{1}-\frac{4}{3} \frac{\boldsymbol{k}_{1} \boldsymbol{\sigma}_{1} \cdot \boldsymbol{k}_{1}}{\boldsymbol{k}_{1}^{2}+m_{\pi}^{2}}\right) \frac{\boldsymbol{\sigma}_{2} \cdot \boldsymbol{k}_{2}}{\boldsymbol{k}_{2}^{2}+m_{\pi}^{2}} \frac{\boldsymbol{\sigma}_{3} \cdot \boldsymbol{k}_{3}}{\boldsymbol{k}_{3}^{2}+m_{\pi}^{2}}
\end{aligned}
$$

where

$$
\sum_{\text {cycle }(l m n)} f_{l m n} \equiv f_{l m n}+f_{m n l}+f_{n l m}
$$
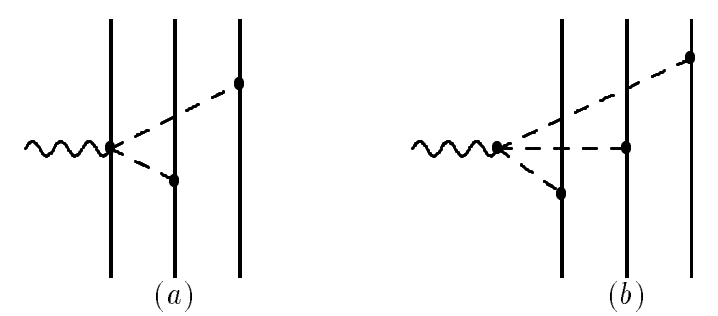

FIG. 4. Diagrams for the three-body GT operator. All other diagrams are higher order than $\mathrm{N}^{4} \mathrm{LO}$ except for the crossed diagrams of $(a)$ - crossed with respect to the particle indices.

\section{Comparison with SNPA exchange currents}

The meson-exchange currents in SNPA [52,53 are based on one-boson exchange diagrams involving those bosons which are responsible for the phenomenological nuclear forces in the context of one-boson-exchange models. This framework does not have direct contact with chiral counting. We give here a detailed comparison between the transition operators used in SNPA and those dictated by $\chi \mathrm{PT}$. Among the most elaborate SNPA operators are the ones used in CRSW91 [16]; these operators were derived by Towner [53 based on a phenomenological Lagrangian [54] which satisfies CVC, PCAC and current algebra. We consider the SNPA operators used in CRSW91 as a representative. It will turn out that there are substantial differences between the SNPA and $\chi \mathrm{PT}$ operators in both the long-range and short-range parts.

In CRSW91 the heavy particles, $\rho$ and $\Delta$, are treated as explicit degrees of freedom.13 To examine the roles of these heavy particles in the context of the present comparison, we divide the two-body currents in CRSW91 into two families:

$$
\begin{aligned}
\boldsymbol{A}^{a}= & \boldsymbol{A}_{I}^{a}+\boldsymbol{A}_{I I}^{a} \\
\equiv & {\left[\boldsymbol{A}^{a}(\Delta \pi)+\boldsymbol{A}^{a}(\pi \rho)+\boldsymbol{A}^{a}(\pi S)\right] } \\
& +\left[\boldsymbol{A}^{a}(\Delta \rho)+\boldsymbol{A}^{a}(\rho S)\right],
\end{aligned}
$$

13 The one-body operators are not sensitive to these additional ingredients as long as the single-nucleon parameters are determined empirically. We note, however, that CRSW91 includes only the leading-order GT operator, $\frac{\tau^{a}}{2} g_{A} \boldsymbol{\sigma}$, without taking into account relativistic corrections. 
where the "S" stands for "seagull". $\boldsymbol{A}_{I}^{a}$ and $\boldsymbol{A}_{I I}^{a}$ can be associated, respectively, with $\boldsymbol{A}_{l m}^{a}(1 \pi)$ and $\boldsymbol{A}_{l m}^{a}(2 \pi)$ in Eq. A.4. The expression for $\boldsymbol{A}_{I}^{a}$ is

$$
\begin{aligned}
& \boldsymbol{A}_{I}^{a}=\frac{g_{A}}{2 m_{N} f_{\pi}^{2}}\{ \\
& -\frac{4}{25} g_{A}^{2} I_{1} \frac{m_{N}}{m_{\Delta}-m_{N}} \mathcal{R}_{\pi}^{2}\left(\boldsymbol{k}_{2}\right)\left[4 \tau_{2}^{a} \boldsymbol{k}_{2}-\left(\vec{\tau}_{1} \times \vec{\tau}_{2}\right)^{a} \boldsymbol{\sigma}_{1} \times \boldsymbol{k}_{2}\right] \\
& -\frac{I_{2}}{4} \mathcal{R}_{\rho}\left(\boldsymbol{k}_{1}\right) \mathcal{R}_{\pi}\left(\boldsymbol{k}_{2}\right) \frac{m_{\rho}^{2}}{m_{\rho}^{2}+\boldsymbol{k}_{1}^{2}}\left(\vec{\tau}_{1} \times \vec{\tau}_{2}\right)^{a}\left[(1+\kappa) \boldsymbol{\sigma}_{1} \times \boldsymbol{k}_{1}-2 i \overline{\boldsymbol{p}}_{1}\right] \\
& +\frac{I_{1}}{4} g_{A}^{2} \mathcal{R}_{\pi}^{2}\left(\boldsymbol{k}_{2}\right)\left[\left(\vec{\tau}_{1} \times \vec{\tau}_{2}\right)^{a} \boldsymbol{\sigma}_{1} \times \boldsymbol{k}_{2}-\tau_{2}^{a}\left(-\boldsymbol{q}+2 i \boldsymbol{\sigma}_{1} \times \overline{\boldsymbol{p}}_{1}\right)\right] \\
& \} \frac{\boldsymbol{\sigma}_{2} \cdot \boldsymbol{k}_{2}}{m_{\pi}^{2}+\boldsymbol{k}_{2}^{2}}+(1 \leftrightarrow 2)
\end{aligned}
$$

with

$$
\mathcal{R}_{\pi}(\boldsymbol{k}) \equiv \frac{\Lambda_{\pi}^{2}-m_{\pi}^{2}}{\Lambda_{\pi}^{2}+\boldsymbol{k}^{2}}, \quad \mathcal{R}_{\rho}(\boldsymbol{k}) \equiv \frac{\Lambda_{\rho}^{2}-m_{\rho}^{2}}{\Lambda_{\rho}^{2}+\boldsymbol{k}^{2}}
$$

where $m_{\Delta} \simeq 1232 \mathrm{MeV}, \kappa \simeq 6.6$, and $g_{\rho} \simeq 2.50$ is the $\rho N N$ coupling constant; $\Lambda_{\pi}\left(\Lambda_{\rho}\right)$ is a cutoff parameter characterizing the $\pi N N(\rho N N)$ coupling form factor. We have defined $I_{1}$ and $I_{2}$ by

$I_{1} \equiv \frac{4 f_{\pi}^{2} f_{\pi N N}^{2}}{g_{A}^{2} m_{\pi}^{2}}=\frac{f_{\pi N N}^{2}}{m_{\pi}^{2}} \cdot\left(\frac{g_{A}^{2}}{4 f_{\pi}^{2}}\right)^{-1}, \quad I_{2} \equiv \frac{8 g_{\rho}^{2} f_{\pi}^{2}}{m_{\rho}^{2}}$

We note that $I_{1}=1$ if we assume the GoldbergerTreiman relation, and $I_{2}=1$ if the KSRF relation holds. ${ }^{15}$ The above equation should be compared with $\boldsymbol{A}_{l m}^{a: \nu 3}(1 \pi)$ in Eq. A.6). A little exercise shows that, while the currents $\boldsymbol{A}(\pi \Delta)$ and $\boldsymbol{A}(\pi \rho)$ can be related to certain currents in $\chi \mathrm{PT}, \boldsymbol{A}(\pi S)$ has no $\chi \mathrm{PT}$ counterpart to the order considered here. A possible explanation for the occurrence of this "extra" term in SNPA is that $\boldsymbol{A}(\pi S)$ arises as a "recoil" term associated with the use of the pseudoscalar coupling. A $\chi \mathrm{PT}$ analog of $\boldsymbol{A}(\pi S)$ would be a $1 / m_{N}$ term, but this term should be substantially suppressed; hence a term like $\boldsymbol{A}(\pi S)$ should be absent in chirally invariant theory. Comparison of the coefficients of $\left(\vec{\tau}_{1} \times \vec{\tau}_{2}\right)^{a} \overline{\boldsymbol{p}}_{1}, \tau_{2}^{a} \boldsymbol{k}_{2},\left(\vec{\tau}_{1} \times \vec{\tau}_{2}\right)^{a} \boldsymbol{\sigma}_{1} \times \boldsymbol{k}_{2}$ and $\left(\vec{\tau}_{1} \times \vec{\tau}_{2}\right)^{a} \boldsymbol{\sigma}_{1} \times \boldsymbol{q}$ leads to the following correspondence between $\chi \mathrm{PT}$ (lefthand side) and CRSW91 (right-hand side):

14 The pseudovector $\pi N N$ coupling constant $f_{\pi N N}$ used in CRSW91 is related to the quantities used here as

$$
\frac{f_{\pi N N}}{m_{\pi}}=\frac{g_{\pi N N}}{2 m_{N}}=\frac{g_{A}}{2 f_{\pi}} .
$$

For the last equality, the Goldberger-Treiman relation has been used.

${ }^{15}$ Since $g_{\rho}$ used in CRSW91 is half of the conventional one, the KSRF relation here reads $2\left(2 g_{\rho}\right)^{2} f_{\pi}^{2}=m_{\rho}^{2}$, rather than $2 g_{\rho}^{2} f_{\pi}^{2}=m_{\rho}^{2}$.

$$
\begin{aligned}
1 & \leftrightarrow I_{2} \mathcal{R}_{\rho}\left(\boldsymbol{k}_{1}\right) \mathcal{R}_{\pi}\left(\boldsymbol{k}_{2}\right) \frac{m_{\rho}^{2}}{m_{\rho}^{2}+\boldsymbol{k}_{1}^{2}}, \\
\hat{c}_{3} \leftrightarrow & -\frac{8}{25} g_{A}^{2} I_{1} \frac{m_{N}}{m_{\Delta}-m_{N}} \mathcal{R}_{\pi}^{2}\left(\boldsymbol{k}_{j}\right), \\
\hat{c}_{4}+\frac{1}{4} \leftrightarrow & \frac{4}{25} g_{A}^{2} I_{1} \frac{m_{N}}{m_{\Delta}-m_{N}} \mathcal{R}_{\pi}^{2}\left(\boldsymbol{k}_{j}\right) \\
& +I_{2} \mathcal{R}_{\rho}\left(\boldsymbol{k}_{1}\right) \mathcal{R}_{\pi}\left(\boldsymbol{k}_{2}\right) \frac{m_{\rho}^{2}}{m_{\rho}^{2}+\boldsymbol{k}_{1}^{2}} \frac{1+\kappa}{4}, \\
1+c_{6} \leftrightarrow & I_{2} \mathcal{R}_{\rho}\left(\boldsymbol{k}_{1}\right) \mathcal{R}_{\pi}\left(\boldsymbol{k}_{2}\right) \frac{m_{\rho}^{2}}{m_{\rho}^{2}+\boldsymbol{k}_{1}^{2}}(1+\kappa) .
\end{aligned}
$$

The presence of the momentum-dependence in $\mathcal{R}$ 's and the $\rho$-meson propagator prevents us from going beyond this correspondence. To proceed, however, we may consider the approximation

$$
1 \approx I_{2} \mathcal{R}_{\rho}\left(\boldsymbol{k}_{1}\right) \mathcal{R}_{\pi}\left(\boldsymbol{k}_{2}\right) \frac{m_{\rho}^{2}}{m_{\rho}^{2}+\boldsymbol{k}_{1}^{2}} \approx I_{1} \mathcal{R}_{\pi}^{2}\left(\boldsymbol{k}_{j}\right)
$$

We then find

$$
\begin{aligned}
\hat{c}_{3}^{\mathrm{CRSW}} & =-\frac{8}{25} g_{A}^{2} \frac{m_{N}}{m_{\Delta}-m_{N}} \simeq-1.633, \\
\hat{c}_{4}^{\mathrm{CRSW}} & =-\frac{1}{2} \hat{c}_{3}^{\mathrm{CRSW}}+\frac{\kappa}{4} \simeq 2.467, \\
c_{6}^{\mathrm{CRSW}} & =\kappa \simeq 6.6 .
\end{aligned}
$$

It is informative to compare these values of $\hat{c}$ 's with those obtained in a resonance-exchange saturation analysis by Bernard, Kaiser and Meissner (BKM) 26]. We note that the two approaches give very different results for the $\Delta$ resonance contributions. CRSW91 used the quark model value for the ratio, $g_{A N \Delta} / g_{\pi N \Delta}$, the accuracy of which is rather difficult to assess. Meanwhile, the resonancesaturation calculation suffers from ambiguity related to the so-called off-shell parameter $Z$. Considering these uncertainties, it is perhaps not too surprising that BKM's estimate of the $\Delta$ contribution to $\hat{c}_{3},\left|\hat{c}_{3}^{\Delta}\right|=3.59$, is 2.2 times larger than the estimate in CRSW91. We also note that, while CRSW91 only includes the $\Delta$ and $\rho$-meson contributions, BKM's calculation contains the contributions from scalar-meson and Roper exchanges as well. According to BMK,

$$
\begin{aligned}
& \hat{c}_{3}^{\Delta}+\hat{c}_{3}^{\text {scalar }}+\hat{c}_{3}^{\text {Roper }}=-3.59-1.31-0.06=-4.96, \\
& \hat{c}_{4}^{\Delta}+\hat{c}_{4}^{\rho}+\hat{c}_{4}^{\text {Roper }}=1.80+1.53+0.11=3.44 . \quad(\mathrm{A}
\end{aligned}
$$

Thus the contributions of the scalar-meson exchange are substantial. What is significant for our calculation is the fact that the coefficients $c$ 's can be extracted directly from the $\pi N$ scattering data [26,51]. The most recent analysis 51] gives

$$
\begin{aligned}
& \hat{c}_{3}=(-5.58 \pm 0.08,-5.49 \pm 0.01,-5.82 \pm 0.08), \\
& \hat{c}_{4}=(3.26 \pm 0.05,3.29 \pm 0.01,3.30 \pm 0.04)
\end{aligned}
$$


These values are in reasonable agreement with those obtained in the resonance saturation approach. We should note, however, that the results in Eq. A.34) belong to an $\mathrm{N}^{4} \mathrm{LO}$ calculation wherein $\Delta(1232)$ as well as other massive degrees of freedom have been integrated out. The explicit inclusion of $\Delta(1232)$ would modify the values of $\hat{c}$ 's, because the $\Delta$ contribution to the $\hat{c}$ 's should now be excluded. We also should pay attention to a similar modification of the LECs as we move from $\mathrm{N}^{4} \mathrm{LO}$ to $\mathrm{N}^{3} \mathrm{LO}$. Although the difference between $\hat{c}$ 's obtained in an $\mathrm{N}^{3} \mathrm{LO}$ calculation and those obtained in $\mathrm{N}^{4} \mathrm{LO}$ are of order of $Q^{4}$ and hence can in principle be neglected in an $\mathrm{N}^{3} \mathrm{LO}$ calculation, it is more natural and safer to use in our present calculation the values obtained in an $\mathrm{N}^{3} \mathrm{LO}$ analysis [26],

$$
\begin{aligned}
\mathrm{N}^{3} \mathrm{LO}: & \hat{c}_{3}=-4.96 \pm 0.23, \\
& \hat{c}_{4}=3.40 \pm 0.09 .
\end{aligned}
$$

The precise value of $c_{6}$ is unimportant in the present context, since it is suppressed by the kinematic factor $|\boldsymbol{q}|$. In any event, the results of BMK and CRSW91 are close to each other, $c_{6} \simeq 5.83$.

We now discuss the short-ranged currents, $\boldsymbol{A}_{I I}^{a}=$ $\boldsymbol{A}(\rho \Delta)+\boldsymbol{A}(\rho S)$. According to CRSW91, the dominant term in $\boldsymbol{A}_{I I}^{a}$ is of the form

$$
\begin{aligned}
& \boldsymbol{A}^{a}(\rho \Delta)=\frac{g_{A}}{2 m_{N} f_{\pi}^{2}} I_{2} \frac{(1+\kappa)^{2}}{50 m_{N}\left(m_{\Delta}-m_{N}\right)} \mathcal{R}_{\rho}^{2}\left(\boldsymbol{k}_{2}\right) \frac{m_{\rho}^{2}}{m_{\rho}^{2}+\boldsymbol{k}_{2}^{2}} \\
& \otimes\left[4 \tau_{2}^{a}\left(\boldsymbol{\sigma}_{2} \times \boldsymbol{k}_{2}\right) \times \boldsymbol{k}_{2}-\left(\vec{\tau}_{1} \times \vec{\tau}_{2}\right)^{a} \boldsymbol{\sigma}_{1} \times\left[\left(\boldsymbol{\sigma}_{2} \times \boldsymbol{k}_{2}\right) \times \boldsymbol{k}_{2}\right]\right] \\
& +(1 \leftrightarrow 2) .
\end{aligned}
$$

It should be noted, however, that this term belongs to $\mathrm{N}^{5} \mathrm{LO}$ in chiral counting, and therefore its inclusion in CRSW91 constitutes a deviation from $\chi$ PT. Although a particular $\mathrm{N}^{5} \mathrm{LO}$ term may give an appreciable contribution (see below), there are many terms of the same order, including multi-loop diagrams, and in general there should be a substantial cancellation among these terms to make the net $\mathrm{N}^{5} \mathrm{LO}$ contribution small, as dictated by chiral symmetry. Thus, there are important differences between $\boldsymbol{A}_{I I}^{a}$ of CRSW91 and $\boldsymbol{A}(2 \pi)$ derived in $\chi \mathrm{PT}$.

\section{APPENDIX B: AXIAL CHARGE OPERATORS}

As stressed in the main text, the axial charge operators are chiral-protected. Since the axial-charge operators up to $\mathcal{O}\left(Q^{4}\right)$ have already been described in detail in Ref. [15], we only briefly recapitulate what is directly relevant to the present work. The leading one-body $A^{0}$ operator is kinematically suppressed because of the $\gamma_{5}$ matrix. Correspondingly, in $\chi \mathrm{PT}$, the $A^{0}$ operator at order $\mathcal{O}\left(Q^{1}\right)$ appears as a $1 / m_{N}$ term. The leading correction to the one-body axial-charge operator comes from the soft one-pion-exchange, which is of $\mathcal{O}\left(Q^{2}\right)$. Loop contributions start at $\mathcal{O}\left(Q^{4}\right)$, and hence the ratio of the loop contribution to the tree diagram two-body contribution is $\mathcal{O}\left(Q^{2}\right)$. Finally, there is no three-body contribution up to $\mathcal{O}\left(Q^{4}\right)$.

The one-body axial-charge operator at threshold is given by

$$
A_{l}^{0, a}=-\frac{\tau_{l}^{a}}{2} g_{A}\left[\frac{\boldsymbol{\sigma}_{l} \cdot \overline{\boldsymbol{p}}_{l}}{m_{N}}+\mathcal{O}\left(\frac{\boldsymbol{q}^{2}}{m_{N}^{2}}\right)\right]
$$

which is $\mathcal{O}\left(Q^{1}\right)$. We note that there is no relativistic correction of $\mathcal{O}(\boldsymbol{q})$ to the one-body axial-charge; this aspect is in sharp contrast to the GT operator.

The two-body $A^{0}$ current appears at $\mathcal{O}\left(Q^{2}\right)$ (tree diagram) and at $\mathcal{O}\left(Q^{4}\right)$ (loop diagrams):

$$
A_{12}^{0, a}=-\frac{g_{A}}{4 f_{\pi}^{2}}\left[\mathcal{T}^{a(I)} \mathcal{W}^{(I)}+\mathcal{T}^{a(I I)} \mathcal{W}^{(I I)}\right]
$$

where

$$
\begin{gathered}
\mathcal{T}^{a(I)}=i\left(\vec{\tau}_{1} \times \vec{\tau}_{2}\right)^{a} \boldsymbol{k} \cdot\left(\boldsymbol{\sigma}_{1}+\boldsymbol{\sigma}_{2}\right), \\
\mathcal{T}^{a(I I)}=i\left(\vec{\tau}_{1}+\vec{\tau}_{2}\right)^{a} \boldsymbol{k} \cdot \boldsymbol{\sigma}_{1} \times \boldsymbol{\sigma}_{2},
\end{gathered}
$$

with $\boldsymbol{k}=\boldsymbol{k}_{2}=-\boldsymbol{k}_{1}$. The one-pion-exchange contribution including the vertex renormalization (loop corrections to the vertices) reads

$$
\begin{aligned}
\mathcal{W}_{1 \pi}^{(I)} & =-\frac{1}{m_{\pi}^{2}-t} F_{1}^{V}(t), \\
\mathcal{W}_{1 \pi}^{(I I)} & =0
\end{aligned}
$$

where $t \equiv k_{0}^{2}-\boldsymbol{k}^{2} \simeq-\boldsymbol{k}^{2}$, and $F_{1}^{V}(t)$ is the isovector Dirac form factor of the nucleon electromagnetic current. The phenomenologically determined $F_{1}^{V}(t)$ is of the dipole type

$$
F_{1}^{V}(t)=\left(\frac{\Lambda^{2}}{\Lambda^{2}-t}\right)^{2}
$$

with $\Lambda=840 \mathrm{MeV}$. The HB $\chi \mathrm{PT}$ expression for $F_{1}^{V}(t)$ up to one-loop accuracy is given by

$$
\begin{aligned}
& F_{1}^{V}(t)=1+\frac{c_{3}^{R}}{f_{\pi}^{2}} t \\
& -\frac{t}{16 \pi^{2} f_{\pi}^{2}}\left[\frac{1+3 g_{A}^{2}}{2} K_{0}(t)-2\left(1+2 g_{A}^{2}\right) K_{2}(t)\right] .
\end{aligned}
$$

The loop functions, $K$ 's, will be specified below. The constant $c_{3}^{R}$ is determined by the nucleon charge radius, ${ }^{16}$

\footnotetext{
16 Note that this $c_{3}^{R}$ has nothing to do with the $c$ 's that appear in the GT operators. This confusing situation arises due to the lack of a unified system for labelling the coefficients
} 


$$
c_{3}^{R} \frac{m_{\pi}^{2}}{f_{\pi}^{2}}=\frac{m_{\pi}^{2}}{6}\left\langle r^{2}\right\rangle_{1}^{V} \simeq 0.04784
$$

We should mention here that $\mathcal{M}_{1 \pi}$ in Eq. B.5 contains both $\mathcal{O}\left(Q^{2}\right)$ and $\mathcal{O}\left(Q^{4}\right)$ contributions. The $\mathcal{O}\left(Q^{2}\right)$ contributions can be obtained by replacing $F_{1}^{V}(t)$ by 1 , while $\left(F_{1}^{V}(t)-1\right)$ is responsible for the $\mathcal{O}\left(Q^{4}\right)$ contributions. The two-pion-exchange contributions, which are also of $\mathcal{O}\left(Q^{4}\right)$, are given by :

$$
\begin{aligned}
\mathcal{W}_{2 \pi}^{(I)}= & \frac{1}{16 \pi^{2} f_{\pi}^{2}}\left[-\frac{3 g_{A}^{2}-2}{4} K_{0}\left(k^{2}\right)-\frac{1}{2} g_{A}^{2} K_{1}\left(k^{2}\right)\right] \\
& -\frac{1}{4 f_{\pi}^{2}} \kappa_{4}^{(1)} \\
\mathcal{W}_{2 \pi}^{(I I)}= & \frac{1}{16 \pi^{2} f_{\pi}^{2}}\left[2 g_{A}^{2} K_{0}\left(k^{2}\right)\right]-\frac{1}{4 f_{\pi}^{2}} \kappa_{4}^{(2)}
\end{aligned}
$$

where $\kappa_{4}^{(1)}$ and $\kappa_{4}^{(2)}$ are unknown parameters 17 . The total two-body axial-charge operator is the sum of Eqs. (B.5) and (B.9):

$$
\begin{gathered}
\mathcal{W}^{(I)}=\mathcal{W}_{1 \pi}^{(I)}+\mathcal{W}_{2 \pi}^{(I)} \\
\mathcal{W}^{(I I)}=\mathcal{W}_{1 \pi}^{(I I)}+\mathcal{W}_{2 \pi}^{(I I)}
\end{gathered}
$$

The loop functions $K$ 's in the above are defined as

$$
\begin{aligned}
& K_{0}(t)=\int_{0}^{1} d z \ln \left[1-z(1-z) \frac{t}{m_{\pi}^{2}}\right], \\
& K_{1}(t)=\int_{0}^{1} d z \frac{-z(1-z) t}{m_{\pi}^{2}-z(1-z) t}, \\
& K_{2}(t)=\int_{0}^{1} d z z(1-z) \ln \left[1-z(1-z) \frac{t}{m_{\pi}^{2}}\right] .
\end{aligned}
$$

The integrations over $z$ can be done analytically, resulting in

$$
\begin{aligned}
& K_{0}(t)=-2+\sigma \ln \left(\frac{\sigma+1}{\sigma-1}\right), \\
& K_{1}(t)=1-\frac{\sigma^{2}-1}{2 \sigma} \ln \left(\frac{\sigma+1}{\sigma-1}\right), \\
& K_{2}(t)=-\frac{4}{9}+\frac{\sigma^{2}}{6}+\frac{\sigma\left(3-\sigma^{2}\right)}{12} \ln \left(\frac{\sigma+1}{\sigma-1}\right),
\end{aligned}
$$

in the chiral Lagrangian. In a chiral expansion $\mathcal{L}=\mathcal{L}_{0}+$ $\mathcal{L}_{1}+\mathcal{L}_{2}+\cdots$, it was once common to use the letter $b(c)$ to label the coefficients appearing in $\mathcal{L}_{1}\left(\mathcal{L}_{2}\right)$. Later, however, it has become more common to use the letter $c$ to designate the coefficients that feature in $\mathcal{L}_{1}$, resulting in unfortunate confusions. In short, the above $c_{3}^{R}$ is a coefficient in $\mathcal{L}_{2}$, while the $c$ 's appearing in the expression for the GT operator belong to $\mathcal{L}_{1}$.

${ }^{17}$ In fact, these are the only unknown parameters up to $\mathrm{N}^{4} \mathrm{LO}$. It turns out that due to the chiral filter mechanism, one can drop these terms when working to $\mathrm{N}^{4} \mathrm{LO}$ for the GT operator. with

$$
\sigma \equiv\left(\frac{4 m_{\pi}^{2}-t}{-t}\right)^{\frac{1}{2}}
$$

\section{APPENDIX C: REGULARIZATION AND THE CUTOFF}

\section{Fourier transform}

Since SNPA employs coordinate-space representation, we need to Fourier transform the momentum-space expressions. In doing so, we must impose a cutoff to regularize the integral. The cutoff introduced here typically represents a scale that divides the low-energy degrees of freedom (which we choose to include explicitly) and the high-energy degrees of freedom (which we integrate out). How to implement cutoff into the theory is not unique, but physics should be independent of methods used insofar as the calculation is done consistently. This is a statement of renormalization group invariance. We now describe a particular cutoff scheme to be used here.18 For the $n_{B}$-body current in momentum space, $\boldsymbol{A}_{12 \cdots n}^{a}=\boldsymbol{A}_{12 \cdots n}^{a}\left(\boldsymbol{k}_{1}, \boldsymbol{k}_{2}, \cdots, \boldsymbol{k}_{n}\right)$, define its "Fourier transform" as

$$
\begin{aligned}
& \tilde{\boldsymbol{A}}_{12 \cdots n}^{a} \equiv\left[\prod_{l=1}^{n} \int \frac{d^{3} \boldsymbol{k}_{l}}{(2 \pi)^{3}} \mathrm{e}^{i \boldsymbol{k}_{l} \cdot \boldsymbol{r}_{l}} S_{\Lambda}\left(\boldsymbol{k}_{l}^{2}\right)\right] \\
& (2 \pi)^{3} \delta^{(3)}\left(\boldsymbol{q}+\boldsymbol{k}_{1}+\boldsymbol{k}_{2}+\cdots+\boldsymbol{k}_{n}\right) \boldsymbol{A}_{12 \cdots n}^{a},
\end{aligned}
$$

where $S_{\Lambda}\left(\boldsymbol{k}^{2}\right)$ is a regulator with a cutoff $\Lambda$, and the factor $(2 \pi)^{3} \delta^{(3)}\left(\boldsymbol{q}+\boldsymbol{k}_{1}+\boldsymbol{k}_{2}+\cdots+\boldsymbol{k}_{n}\right)$ comes from momentum conservation. We employ here a regulator of the Gaussian type 19

$$
S_{\Lambda}\left(\boldsymbol{k}^{2}\right)=\exp \left(-\frac{\boldsymbol{k}^{2}}{2 \Lambda^{2}}\right) .
$$

For a one-body operator, the regulator plays no role, see Eq.(A.3). Now for the two-body current, Eq.(C.1) gives 20

${ }^{18}$ We illustrate our method for $\boldsymbol{A}$, but the same method is used for the other currents as well.

${ }^{19}$ Using a Gaussian cutoff can in principle upset the chiral counting even when graphs up to a definite chiral order (say $\nu$ ) are considered. This counting mismatch, however, occurs at an order higher than $\nu$, and furthermore the error committed is likely to be minimized by the regularization scheme we have adopted for the $\hat{d}^{R}$ counter term.

${ }^{20}$ From here on, we shall specialize ourselves to the threshold limit, $\boldsymbol{q}=\mathbf{0}$. 


$$
\begin{aligned}
\tilde{\boldsymbol{A}}_{12}^{a}= & \int \frac{d^{3} \boldsymbol{k}}{(2 \pi)^{3}} S_{\Lambda}^{2}\left(\boldsymbol{k}^{2}\right) \mathrm{e}^{-i \boldsymbol{k} \cdot \boldsymbol{r}_{12}} \\
& \times \boldsymbol{A}_{12}^{a}\left(\boldsymbol{k}_{1}=-\boldsymbol{k}, \boldsymbol{k}_{2}=\boldsymbol{k}\right) .
\end{aligned}
$$

To simplify subsequent expressions, we define the following functions:

$$
\begin{aligned}
\delta_{\Lambda}^{(3)}(\boldsymbol{r}) & \equiv \int \frac{d^{3} \boldsymbol{k}}{(2 \pi)^{3}} S_{\Lambda}^{2}\left(\boldsymbol{k}^{2}\right) \mathrm{e}^{i \boldsymbol{k} \cdot \boldsymbol{r}}, \\
y_{0 \Lambda}(m, r) & \equiv \int \frac{d^{3} \boldsymbol{k}}{(2 \pi)^{3}} S_{\Lambda}^{2}\left(\boldsymbol{k}^{2}\right) \mathrm{e}^{i \boldsymbol{k} \cdot \boldsymbol{r}} \frac{1}{\boldsymbol{k}^{2}+m^{2}}, \\
y_{1 \Lambda}(m, r) & \equiv-\frac{1}{m} \frac{\partial}{\partial r} y_{0 \Lambda}(m, r), \\
y_{2 \Lambda}(m, r) & \equiv \frac{1}{m^{2}} r \frac{\partial}{\partial r} \frac{1}{r} \frac{\partial}{\partial r} y_{0 \Lambda}(m, r) .
\end{aligned}
$$

These functions become the ordinary delta and Yukawa functions when $\Lambda$ goes to infinity. We also use the abbreviation $y_{0 \Lambda}^{\pi}(r) \equiv y_{0 \Lambda}\left(m_{\pi}, r\right)$, and similarly for $y_{1 \Lambda}^{\pi}(r)$ and $y_{2 \Lambda}^{\pi}(r)$. The regularized delta and Yukawa functions read

$$
\begin{aligned}
\delta_{\Lambda}^{(3)}(\boldsymbol{r})= & \frac{\Lambda^{3}}{(4 \pi)^{\frac{3}{2}}} \exp \left(-\frac{\Lambda^{2} r^{2}}{4}\right), \\
y_{0 \Lambda}(m, r)= & \frac{1}{4 \pi r} \mathrm{e}^{\frac{m^{2}}{\Lambda^{2}}} \frac{1}{2}\left[\mathrm{e}^{-m r} \operatorname{erfc}\left(-\frac{\Lambda r}{2}+\frac{m}{\Lambda}\right)\right. \\
& -(r \leftrightarrow-r)] .
\end{aligned}
$$

We are now ready to write down the $\nu=3$ two-body axial-vector current, Eq. A.6. in coordinate space:

$$
\begin{aligned}
& \tilde{\boldsymbol{A}}_{12}^{a: \nu 3}(1 \pi)= \\
& \frac{g_{A}}{2 m_{N} f_{\pi}^{2}} \delta_{\Lambda}^{(3)}\left(\boldsymbol{r}_{12}\right)\left[\frac{1}{3} \hat{c}_{3}\left(\mathcal{O}_{+}^{a}+\mathcal{O}_{-}^{a}\right)+\frac{2}{3}\left(\hat{c}_{4}+\frac{1}{4}\right) \mathcal{O}_{\times}^{a}\right] \\
& -\frac{g_{A} m_{\pi}^{2}}{2 m_{N} f_{\pi}^{2}}\left[\mathcal{O}_{\mathcal{P}}^{a} y_{1 \Lambda}^{\pi}\left(r_{12}\right)\right. \\
& +\left(\hat{c}_{3}\left(\mathcal{T}_{+}^{a}+\boldsymbol{\mathcal { T }}_{-}^{a}\right)-\left(\hat{c}_{4}+\frac{1}{4}\right) \boldsymbol{\mathcal { T }}_{\times}^{a}\right) y_{2 \Lambda}^{\pi}\left(r_{12}\right) \\
& \left.+\left(\frac{1}{3} \hat{c}_{3}\left(\mathcal{O}_{+}^{a}+\mathcal{O}_{-}^{a}\right)+\frac{2}{3}\left(\hat{c}_{4}+\frac{1}{4}\right) \mathcal{O}_{\times}^{a}\right) y_{0 \Lambda}^{\pi}\left(r_{12}\right)\right], \\
& \tilde{\boldsymbol{A}}_{12}^{a: \nu 3}(2 \pi)= \\
& \frac{g_{A}}{2 m_{N} f_{\pi}^{2}} \delta_{\Lambda}^{(3)}\left(\boldsymbol{r}_{12}\right)\left[\hat{d}_{1}\left(\mathcal{O}_{+}^{a}+\mathcal{O}_{-}^{a}\right)+2 \hat{d}_{2} \mathcal{O}_{\times}^{a}\right]
\end{aligned}
$$

where the superscript $(i, j)$ are particle indices, $r_{12} \equiv$ $\left|\boldsymbol{r}_{12}\right|$ and $\hat{\boldsymbol{r}}_{12} \equiv \frac{\boldsymbol{r}_{12}}{r_{12}}$. In the above equations, we have defined the following two-body spin-isospin operators

$$
\begin{aligned}
& \mathcal{O}_{\odot}^{i, a} \equiv\left(\vec{\tau}_{1} \odot \vec{\tau}_{2}\right)^{a}\left(\boldsymbol{\sigma}_{1} \odot \boldsymbol{\sigma}_{2}\right)^{i}, \\
& \mathcal{T}_{\odot}^{i, a} \equiv\left(\hat{r}_{12}^{i} \hat{r}_{12}^{j}-\frac{\delta^{i j}}{3}\right) \mathcal{O}_{\odot}^{a j}
\end{aligned}
$$

where $\odot=(+,-, \times)$ and

$$
\mathcal{O}_{\mathcal{P}}^{i, a} \equiv-\frac{1}{2 m_{\pi}}\left(\vec{\tau}_{1} \times \vec{\tau}_{2}\right)^{a}\left(\bar{p}_{1}^{i} \boldsymbol{\sigma}_{2} \cdot \hat{\boldsymbol{r}}_{12}+\bar{p}_{2}^{i} \boldsymbol{\sigma}_{1} \cdot \hat{\boldsymbol{r}}_{12}\right) .
$$

Note that $\mathcal{O}_{\mathcal{P}}^{i, a}$ is completely determined by Lorentz invariance. In terms of these seven operators, we can write all the two-body currents (including $\nu=3$ and $\nu=4$ contributions) as

$$
\begin{aligned}
\tilde{A}_{12}^{i, a}= & -\sum_{\odot=+,-, \times}\left[F_{\odot}^{C}\left(r_{12}\right) \mathcal{O}_{\odot}^{i, a}+F_{\odot}^{T}\left(r_{12}\right) \mathcal{T}_{\odot}^{i, a}\right] \\
& -\frac{g_{A} m_{\pi}^{2}}{2 m_{N} f_{\pi}^{2}} y_{1 \Lambda}^{\pi}\left(r_{12}\right) \mathcal{O}_{\mathcal{P}}^{i, a} \\
& +\frac{g_{A}}{2 m_{N} f_{\pi}^{2}} \delta_{\Lambda}^{(3)}\left(\boldsymbol{r}_{12}\right)\left[\sum_{\odot=+,-, \times} \hat{d}_{\odot} \mathcal{O}_{\odot}^{i, a}\right] .
\end{aligned}
$$

We have separated out here the part proportional to $\delta_{\Lambda}^{(3)}(\boldsymbol{r})$. The dimensionless parameters $\hat{d}_{\odot}$ are given by $\hat{d}_{1,2}$ and (higher order) loop contributions as

$$
\begin{aligned}
\hat{d}_{+} \equiv & \hat{d}_{1}+\frac{1}{3} \hat{c}_{3} \\
& -\frac{g_{A}^{2} m_{N} m_{\pi}}{32 \pi}\left(-3 X_{1}+9 X_{\sigma \tau}+9 X_{\sigma}-3 X_{\tau}\right), \\
\hat{d}_{-} \equiv & \hat{d}_{1}+\frac{1}{3} \hat{c}_{3} \\
& -\frac{g_{A}^{2} m_{N} m_{\pi}}{32 \pi}\left(-3 X_{1}+9 X_{\sigma \tau}-9 X_{\sigma}+3 X_{\tau}\right), \\
\hat{d}_{\times} \equiv & 2\left[\hat{d}_{2}+\frac{1}{3}\left(\hat{c}_{4}+\frac{1}{4}\right)+\frac{g_{A}^{2} m_{N} m_{\pi}}{32 \pi} X_{\sigma \tau}\right] .
\end{aligned}
$$

The functions $F_{\odot}^{C, T}$ in Eq. (C.11) are given by

$$
\begin{aligned}
F_{+}^{C}(r)= & \frac{g_{A} m_{\pi}^{2}}{2 m_{N} f_{\pi}^{2}} \frac{\hat{c}_{3}^{R}}{3} y_{0 \Lambda}^{\pi}(r) \\
+ & \frac{g_{A}^{3}}{32 \pi f_{\pi}^{4}}\left\{\frac{1}{6}\left(3 D_{1}+k^{2} D_{2}-\tilde{D}_{1 \pi}\right) \frac{m_{\pi}^{2}}{m_{\pi}^{2}+k^{2}}\right. \\
& +\frac{1}{8}\left(3 D_{1}+k^{2} D_{2}\right) \\
& \left.+\frac{g_{A}^{2}}{64}\left(3 D_{3}-k^{2} D_{4}+2 k^{2} D_{5}\right)\right\}_{\mathrm{FT}}(r), \\
F_{-}^{C}(r)= & \frac{g_{A} m_{\pi}^{2}}{2 m_{N} f_{\pi}^{2}} \frac{\hat{c}_{3}^{R}}{3} y_{0 \Lambda}^{\pi}(r) \\
+ & \frac{g_{A}^{3}}{32 \pi f_{\pi}^{4}}\left\{\frac{1}{6}\left(3 D_{1}+k^{2} D_{2}-\tilde{D}_{1 \pi}\right) \frac{m_{\pi}^{2}}{m_{\pi}^{2}+k^{2}}\right. \\
& +\frac{1}{24}\left(3 D_{1}+k^{2} D_{2}\right) \\
& \left.+\frac{g_{A}^{2}}{64}\left(-D_{3}+\frac{1}{3} k^{2} D_{4}+\frac{2}{3} k^{2} D_{5}\right)\right\}_{\mathrm{FT}}(r), \\
F_{\times}^{C}(r)= & \frac{g_{A} m_{\pi}^{2}}{2 m_{N} f_{\pi}^{2}} \frac{2}{3}\left(\hat{c}_{4}^{R}+\frac{1}{4}\right) y_{0 \Lambda}^{\pi}(r)+\frac{g_{A}^{3}}{32 \pi f_{\pi}^{4}} \\
& \otimes\left\{\frac{2}{3} D_{1}-\frac{2}{3}\left(D_{1}-D_{1 \pi}\right) \frac{m_{\pi}^{2}}{m_{\pi}^{2}+k^{2}}\right\}_{\mathrm{FT}}(r)
\end{aligned}
$$


and

$$
\begin{aligned}
F_{+}^{T}(r)= & \frac{g_{A} m_{\pi}^{2}}{2 m_{N} f_{\pi}^{2}} \hat{c}_{3}^{R} y_{2 \Lambda}^{\pi}(r) \\
+ & \frac{g_{A}^{3}}{32 \pi f_{\pi}^{4}}\left\{\frac{1}{2}\left(3 D_{1}+k^{2} D_{2}-\tilde{D}_{1 \pi}\right) \frac{1}{m_{\pi}^{2}+k^{2}}\right. \\
& \left.+\frac{1}{4} D_{2}+\frac{g_{A}^{2}}{64}\left(-6 D_{4}+3 D_{5}\right)\right\}_{\mathrm{FT}}(r), \\
F_{-}^{T}(r)= & \frac{g_{A} m_{\pi}^{2}}{2 m_{N} f_{\pi}^{2}} \hat{c}_{3}^{R} y_{2 \Lambda}^{\pi}(r) \\
+ & \frac{g_{A}^{3}}{32 \pi f_{\pi}^{4}}\left\{\frac{1}{2}\left(3 D_{1}+k^{2} D_{2}-\tilde{D}_{1 \pi}\right) \frac{1}{m_{\pi}^{2}+k^{2}}\right. \\
& \left.-\frac{1}{4} D_{2}+\frac{g_{A}^{2}}{64}\left(2 D_{4}+D_{5}\right)\right\}_{\mathrm{FT}}(r), \\
F_{\times}^{T}(r)= & -\frac{g_{A} m_{\pi}^{2}}{2 m_{N} f_{\pi}^{2}}\left(\hat{c}_{4}^{R}+\frac{1}{4}\right) y_{2 \Lambda}^{\pi}(r)+\frac{g_{A}^{3}}{32 \pi f_{\pi}^{4}} \\
& \times\left\{\frac{D_{1}-D_{1 \pi}}{m_{\pi}^{2}+k^{2}}\right\}_{\mathrm{FT}}^{T}(r),
\end{aligned}
$$

where $k \equiv|\boldsymbol{k}|, D_{i}=D_{i}(k)$ and

$$
\begin{aligned}
& \left\{f\left(k^{2}\right)\right\}_{\mathrm{FT}}(r) \equiv \int \frac{d^{3} \boldsymbol{k}_{2}}{(2 \pi)^{3}} S_{\Lambda}^{2}\left(\boldsymbol{k}^{2}\right) \mathrm{e}^{-i \boldsymbol{k} \cdot \boldsymbol{r}} f\left(k^{2}\right), \\
& \left\{f\left(k^{2}\right)\right\}_{\mathrm{FT}}^{T}(r) \equiv r \frac{\partial}{\partial r} \frac{1}{r} \frac{\partial}{\partial r} \int \frac{d^{3} \boldsymbol{k}_{2}}{(2 \pi)^{3}} S_{\Lambda}^{2}\left(\boldsymbol{k}^{2}\right) \mathrm{e}^{-i \boldsymbol{k} \cdot \boldsymbol{r}} f\left(k^{2}\right) .
\end{aligned}
$$

The explicit results of Fourier transformation of $D_{i}(k)$ and $k^{2} D_{i}(k)$ are given by (" $\rightarrow$ " denotes Fourier transformation):

$$
\begin{aligned}
D_{1} & \rightarrow-\frac{m_{\pi}^{2}}{2 \pi r^{2}} \int_{0}^{1} d z K_{2}(x), \\
D_{2} & \rightarrow \frac{1}{2 \pi r^{2}} \int_{0}^{1} d z z \bar{z} x K_{1}(x), \\
D_{3} & \rightarrow \frac{m_{\pi}^{2}}{2 \pi r^{2}} \int_{0}^{1} d z\left(6 K_{2}(x)+x K_{1}(x)\right), \\
D_{4} & \rightarrow \frac{1}{2 \pi r^{2}} \int_{0}^{1} d z\left[z \bar{z} x^{2} K_{0}(x)+(6 z \bar{z}-1) x K_{1}(x)\right], \\
D_{5} & \rightarrow \frac{1}{2 \pi r^{2}} \int_{0}^{1} d z x K_{1}(x)
\end{aligned}
$$

and

$$
\begin{aligned}
\boldsymbol{k}^{2} D_{2}= & D_{1}-m_{\pi}^{2} D_{5} \rightarrow-\frac{m_{\pi}^{2}}{2 \pi r^{2}} \int_{0}^{1} d z\left(K_{2}(x)+x K_{1}(x)\right), \\
\boldsymbol{k}^{2} D_{4} \rightarrow & -\frac{m_{\pi}^{2}}{2 \pi r^{2}} \int_{0}^{1} d z\left[\frac{6 z \bar{z}-1}{z \bar{z}}\left(2 x K_{1}(x)+K_{2}(x)\right)\right. \\
& \left.+x^{2} K_{0}(x)-x K_{1}(x)\right], \\
\boldsymbol{k}^{2} D_{5} \rightarrow & -\frac{m_{\pi}^{2}}{2 \pi r^{2}} \int_{0}^{1} d z \frac{1}{z \bar{z}}\left(2 x K_{1}(x)+K_{2}(x)\right), \quad \text { (C.16) }
\end{aligned}
$$

where

$$
x \equiv \frac{m_{\pi} r}{\sqrt{z \bar{z}}} .
$$

Next we turn to the three-body currents of Eq. (A.17). The Fourier-transformed three-body current has the form

$$
\begin{aligned}
& \tilde{\boldsymbol{A}}_{123}^{a} \\
& =-\sum_{\text {cycle }(123)} \frac{g_{A}^{3}}{16 f_{\pi}^{4}}\left(2 \tau_{1}^{a} \vec{\tau}_{2} \cdot \vec{\tau}_{3}-\tau_{2}^{a} \vec{\tau}_{3} \cdot \vec{\tau}_{1}-\tau_{3}^{a} \vec{\tau}_{1} \cdot \vec{\tau}_{2}\right) \tilde{\boldsymbol{I}}_{123}
\end{aligned}
$$

with

$$
\begin{aligned}
\tilde{\boldsymbol{I}}_{123} \equiv & {\left[\prod_{i=l}^{3} \int \frac{d^{3} \boldsymbol{k}_{l}}{(2 \pi)^{3}} \mathrm{e}^{i \boldsymbol{k}_{l} \cdot \boldsymbol{r}_{l}} \mathrm{e}^{-\frac{\boldsymbol{k}_{l}^{2}}{2 \Lambda^{2}}}\right] } \\
& \otimes(2 \pi)^{3} \delta^{(3)}\left(\boldsymbol{k}_{1}+\boldsymbol{k}_{2}+\boldsymbol{k}_{3}\right) \\
& \left(\boldsymbol{\sigma}_{1}-\frac{4}{3} \frac{\boldsymbol{k}_{1} \boldsymbol{\sigma}_{1} \cdot \boldsymbol{k}_{1}}{\boldsymbol{k}_{1}^{2}+m_{\pi}^{2}}\right) \frac{\boldsymbol{\sigma}_{2} \cdot \boldsymbol{k}_{2}}{\boldsymbol{k}_{2}^{2}+m_{\pi}^{2}} \frac{\boldsymbol{\sigma}_{3} \cdot \boldsymbol{k}_{3}}{\boldsymbol{k}_{3}^{2}+m_{\pi}^{2}} .
\end{aligned}
$$

The calculation of $\tilde{I}_{123}^{i}$ is rather involved. We may start with exploiting the identity

$$
(2 \pi)^{3} \delta^{(3)}\left(\boldsymbol{k}_{1}+\boldsymbol{k}_{2}+\boldsymbol{k}_{3}\right)=\int d^{3} \boldsymbol{x} \mathrm{e}^{i \boldsymbol{x} \cdot\left(\boldsymbol{k}_{1}+\boldsymbol{k}_{2}+\boldsymbol{k}_{3}\right)}
$$

to arrive at

$$
\begin{array}{r}
\tilde{I}_{123}^{i}=-\frac{4 m_{\pi}^{4}}{3} \int d^{3} \boldsymbol{x}\left[\frac{5}{12 m_{\pi}^{2}} \sigma_{1}^{i} \delta_{\bar{\Lambda}}^{(3)}(\boldsymbol{x})+\sigma_{1}^{i} y_{0 \bar{\Lambda}}^{\pi}(|\boldsymbol{x}|)\right. \\
\left.-\left(\hat{x}^{i} \hat{x}^{j}-\frac{\delta^{i j}}{3}\right) \sigma_{1}^{j} y_{2 \bar{\Lambda}}^{\pi}(|\boldsymbol{x}|)\right] \frac{\boldsymbol{\sigma}_{2} \cdot\left(\boldsymbol{x}+\boldsymbol{r}_{12}\right)}{\left|\boldsymbol{x}+\boldsymbol{r}_{12}\right|} \\
\otimes y_{1 \bar{\Lambda}}^{\pi}\left(\left|\boldsymbol{x}+\boldsymbol{r}_{12}\right|\right) \frac{\boldsymbol{\sigma}_{3} \cdot\left(\boldsymbol{x}+\boldsymbol{r}_{13}\right)}{\left|\boldsymbol{x}+\boldsymbol{r}_{13}\right|} y_{1 \bar{\Lambda}}^{\pi}\left(\left|\boldsymbol{x}+\boldsymbol{r}_{13}\right|\right),
\end{array}
$$

where

$$
\bar{\Lambda} \equiv \sqrt{2} \Lambda .
$$

This representation is nicely transparent, and the resulting integrand is non-oscillatory and rapidly damping. The remaining integration can be done by means of a Monte Carlo simulation with Metropolis random walks.

\section{The parameter $\hat{d}^{R}$}

Up to $\mathrm{N}^{3} \mathrm{LO}$, unknown parameters occur only in $\boldsymbol{A}$. At $\mathrm{N}^{4} \mathrm{LO}$, several unknown parameters appear in both $\boldsymbol{V}$ and $A_{0}$, but no new parameters appear in $\boldsymbol{A}$. By the chiral filter argument, one can ignore the $\mathrm{N}^{4} \mathrm{LO}$ terms in both $\boldsymbol{V}$ and $A_{0}$ while going to $\mathrm{N}^{4} \mathrm{LO}$ in $\boldsymbol{A}$. Thus, up to $\mathrm{N}^{4} \mathrm{LO}$, the only genuinely unknown parameters reside in $\boldsymbol{A}$. The crucial observation is that up to $N^{4} L O$, there is effectively only one constant $\hat{d}^{R}$ that governs the GT amplitudes of all the cases under consideration. The argument goes as follows. 
The two parameters, $\hat{d}_{1}$ and $\hat{d}_{2}$, and the four $X$ 's can be combined into three unknown parameters, $\hat{d}_{ \pm, \times}$, that reflect short-range physics. It is the Fermi-Dirac statistics that reduces the number of unknowns from three to one. To see this, let $\Xi^{\sigma}$ and $\Xi^{\tau}$ be the exchange operators in spin and isospin spaces, respectively; $\Xi^{\sigma}=\frac{1}{2}\left(1+\boldsymbol{\sigma}_{1} \cdot \boldsymbol{\sigma}_{2}\right)$, and $\Xi^{\tau}=\frac{1}{2}\left(1+\vec{\tau}_{1} \cdot \vec{\tau}_{2}\right)$. An explicit calculation gives the identity $\boldsymbol{\sigma}_{1} \times \boldsymbol{\sigma}_{2}=i\left(\boldsymbol{\sigma}_{1}-\boldsymbol{\sigma}_{2}\right) \Xi^{\sigma}$, and likewise for $\vec{\tau}_{1} \times \vec{\tau}_{2}$. Now, the Fermi-Dirac statistics requires that $\Xi^{r} \Xi^{\sigma} \Xi^{\tau}=-1$, where $\Xi^{r}$ is the Majorana exchange operator that exchanges the orbital coordinates, $\boldsymbol{r}_{1}$ and $\boldsymbol{r}_{2}$. As a result,

$$
\mathcal{O}_{\times}^{i, a}=-\mathcal{O}_{-}^{i, a} \Xi^{\sigma} \Xi^{\tau}=\mathcal{O}_{-}^{i, a} \Xi^{r}
$$

When multiplied by the delta function $\delta^{(3)}(\boldsymbol{r})$, the operators are non-vanishing only for the $L=0$ states, which then implies $S+T=1$. Acting on $L=0$ states, $\mathcal{O}_{+}^{i, a}$ is identically zero, since either spin or isospin must be equal to zero. Furthermore, the $L=0$ states are eigenstates of the operator $\Xi^{r}$ with eigenvalue 1 , so that $\mathcal{O}_{-}^{i, a}$ becomes identical to $\mathcal{O}_{x}^{i, a}$. Thus we are left with only one unknown parameter, $\hat{d}^{R} \equiv \hat{d}_{-}+\hat{d}_{\times}$.

The above argument is not strictly valid for the cutoff delta function $\delta_{\Lambda}^{(3)}(\boldsymbol{r})$, which has a finite (albeit very small) range, $\sim \Lambda^{-1}$. However, deviations from the ordinary delta function case is higher order in chiral counting and hence can be ignored.

\section{APPENDIX D: CALCULATION UP TO $\mathrm{N}^{3} \mathrm{LO}$}

We have derived all the weak currents up to $\mathrm{N}^{4} \mathrm{LO}$. As Table indicates, loop contributions start at $\mathrm{N}^{4} \mathrm{LO}$. Loop corrections in the vector currents (both $\boldsymbol{V}$ and $V^{0}$ ) can be safely ignored, since even their leading single-particle terms are suppressed relative to the axial current. It turns out that the loop diagrams in $\boldsymbol{A}$ are all finite and hence need no regularization although there are finite counter terms that should be taken into account. On the other hand, the loop diagrams in $A_{0}$ do have divergences and need to be regularized. To derive the momentum space expressions for the currents given above, we have employed the dimensional regularization. This is not quite congruous with the cutoff regularization adopted in going from momentum to coordinate space. Meanwhile, using a cutoff regularization in evaluating loop graphs is a delicate matter, since that might endanger chiral symmetry; with the use of a cutoff regularization one might need chiral-symmetry-breaking counter terms in order to satisfy the Ward identities. We have not yet investigated whether the dimensional regularization as used here preserves chiral symmetry, and hence we cannot say at this point whether our coordinate space operators at $\mathrm{N}^{4} \mathrm{LO}$ are fully consistent. However, this problem does not arise if we limit ourselves to $\mathrm{N}^{3} \mathrm{LO}$, for up to this order there are no loop contributions. The relevant 2-body currents in coordinate space are:

$$
\begin{aligned}
\boldsymbol{V}_{12}(\boldsymbol{r})= & -\tau_{\times}^{-} \frac{g_{A}^{2} m_{\pi}^{2}}{12 f_{\pi}^{2}} \boldsymbol{r}\left[\boldsymbol{\sigma}_{1} \cdot \boldsymbol{\sigma}_{2} y_{0 \Lambda}^{\pi}(r)+S_{12}(\hat{r}) y_{2 \Lambda}^{\pi}(r)\right] \\
- & i \frac{g_{A}^{2}}{8 f_{\pi}^{2}} \boldsymbol{k} \times\left[\boldsymbol{\mathcal { O }}_{\times} y_{0 \Lambda}^{\pi}(r)+\left(\mathcal{T}_{\times}-\frac{2}{3} \boldsymbol{\mathcal { O }}_{\times}\right) y_{1 \Lambda}^{\pi}(r)\right], \\
A_{12}^{0}(\boldsymbol{r})= & \tau_{\times}^{-} \frac{g_{A}}{4 f_{\pi}^{2}}\left[\boldsymbol{\sigma}_{+} \cdot \hat{r} \frac{y_{1 \Lambda}^{\pi}(r)}{r}+\frac{i}{2} \boldsymbol{k} \cdot \hat{r} \boldsymbol{\sigma}_{-} \cdot \hat{r} y_{1 \Lambda}^{\pi}(r)\right], \\
\boldsymbol{A}_{12}(\boldsymbol{r})= & -\frac{g_{A} m_{\pi}^{2}}{2 m_{N} f_{\pi}^{2}}[ \\
& \left\{\frac{\hat{c}_{3}}{3}\left(\mathcal{O}_{+}+\mathcal{O}_{-}\right)+\frac{2}{3}\left(\hat{c}_{4}+\frac{1}{4}\right) \mathcal{O}_{\times}\right\} y_{0 \Lambda}^{\pi}(r) \\
& \left.+\left\{\hat{c}_{3}\left(\mathcal{T}_{+}+\mathcal{T}_{-}\right)-\left(\hat{c}_{4}+\frac{1}{4}\right) \mathcal{T}_{\times}\right\} y_{2 \Lambda}^{\pi}(r)\right] \\
+ & \frac{g_{A}}{2 m_{N} f_{\pi}^{2} r}\left[\frac{1}{2} \tau_{\times}^{-}\left(\overline{\boldsymbol{p}}_{1} \boldsymbol{\sigma}_{2} \cdot \hat{\boldsymbol{r}}_{+}+\overline{\boldsymbol{p}}_{2} \boldsymbol{\sigma}_{1} \cdot \hat{\boldsymbol{r}}\right) y_{1 \Lambda}^{\pi}(r)\right. \\
& \left.+\delta_{\Lambda}^{(3)}(\boldsymbol{r}) \hat{d}^{R} \mathcal{O}_{\times}\right],
\end{aligned}
$$

with

$$
\left(\delta_{\Lambda}^{(3)}(\boldsymbol{r}), y_{0 \Lambda}^{\pi}(r)\right) \equiv \int \frac{d^{3} \boldsymbol{k}}{(2 \pi)^{3}} \mathrm{e}^{-\frac{\boldsymbol{k}^{2}}{\Lambda^{2}}} \mathrm{e}^{i \boldsymbol{k} \cdot \boldsymbol{r}}\left(1, \frac{1}{\boldsymbol{k}^{2}+m_{\pi}^{2}}\right),
$$

$y_{1 \Lambda}^{\pi}(r) \equiv-r \frac{\partial}{\partial r} y_{0 \Lambda}^{\pi}(r)$ and $y_{2 \Lambda}^{\pi}(r) \equiv \frac{r}{m_{\pi}^{2}} \frac{\partial}{\partial r} \frac{1}{r} \frac{\partial}{\partial r} y_{0 \Lambda}^{\pi}(r)$, where $\mathcal{O}_{\odot}^{k} \equiv \tau_{\odot}^{-} \boldsymbol{\sigma}_{\odot}^{k}, \mathcal{T}_{\odot}^{k} \equiv\left(\hat{r}^{k} \hat{r}^{\ell}-\frac{\delta^{k \ell}}{3}\right) \mathcal{O}_{\odot}^{\ell}$ and

$$
\hat{d}^{R} \equiv \hat{d}_{1}+2 \hat{d}_{2}+\frac{1}{3} \hat{c}_{3}+\frac{2}{3} \hat{c}_{4}+\frac{1}{6} .
$$

The derivative operators, $\overline{\boldsymbol{p}}_{l}(l=1,2)$ in eq.(22), should be understood to act only on the wave functions.

Since $\hat{d}_{R}$ accompanies the regularized $\delta$-function $\delta_{\Lambda}^{(3)}(\boldsymbol{r})$, its contribution depends on $\Lambda$ rather strongly. However, the renormalization-group invariance of EFT requires that this sensitivity to $\Lambda$ should be compensated by the contributions of the remaining terms. Since the single-particle piece of $\boldsymbol{A}$ has no $\Lambda$ dependence, and since all the currents other than $\boldsymbol{A}$ have only weak $\Lambda$ dependence, this compensation must occur between the finiterange two-body GT and the regularized delta-function term. This has been indeed verified in our calculation over a wide range of $\Lambda(500 \mathrm{MeV}-800 \mathrm{MeV})$ although, as mentioned above, the results for the $800 \mathrm{MeV}$ cutoff should be viewed with caution.

[1] For a recent review, see, for example, J. Carlson and R. Schiavilla, Rev. Mod. Phys. 70, 743 (1998).

[2] G.E. Brown and M. Rho, "Double decimation and sliding vacua in the nuclear many-body system," to appear. 
[3] M. Harada and K. Yamawaki, Phys. Rev. D64, 014023 (2001); Phys. Rep., to appear.

[4] G.E. Brown and M. Rho, Phys. Rev. Lett. 66, 2720 (1991).

[5] S.K. Bogner, T.T.S. Kuo, A. Schwenk, D.R. Entem and R. Machleidt, "Towards a unique low momentum nucleon-nucleon interaction", nucl-th/0108041; S.K. Bogner, A. Schwenk, T.T.S. Kuo, and G.E. Brown, "Renormalization group equation for low momentum effective nuclear interactions", nucl-th/0111042.

[6] A. Schwenk, B. Friman, and G.E. Brown, Nucl. Phys. A703, 745 (2002).

[7] T.-S. Park, L.E. Marcucci, R. Schiavilla, M. Viviani, A. Kievsky, S. Rosati, K. Kubodera, D.-P. Min, and M. Rho, nucl-th/0106025.

[8] T.-S. Park, L.E. Marcucci, R. Schiavilla, M. Viviani, A. Kievsky, S. Rosati, K. Kubodera, D.-P. Min, and M. Rho, nucl-th/0107012

[9] J.N. Bahcall, Phys. Rep. 333, 47 (2000), and references therein.

[10] R. Escribano, J.-M. Frere, A. Gevaert, and D. Monderen, Phys. Lett. B444, 397 (1998).

[11] K. Kubodera, J. Delorme, and M. Rho, Phys. Rev. Lett. 40, 755 (1978); M. Rho, Phys. Rev. Lett. 66, 1275 (1991).

[12] G.E. Brown and M. Rho, Phys. Rep. 363, 85 (2002); hep-ph/0103102.

[13] S.M. Ananyan, B.D. Serot and J.D. Walecka, "The axial-vector current in nuclear many-body physics," nuclth/0207019.

[14] T.-S. Park, D.-P. Min, and M. Rho, Phys. Rev. Lett. 74, 4153 (1995); Nucl. Phys. A596, 515 (1996).

[15] T.-S. Park, D.-P. Min, and M. Rho, Phys. Rep. 233, 341 (1993); T.-S. Park, I.S. Towner, and K. Kubodera, Nucl. Phys. A579, 381 (1994).

[16] J. Carlson, D.O. Riska, R. Schiavilla, and R.B. Wiringa, Phys. Rev. C44, 619 (1991).

[17] R. Schiavilla, R.B. Wiringa, V.R. Pandharipande, and J. Carlson, Phys. Rev. C45, 2628 (1992).

[18] L.E. Marcucci, R. Schiavilla, M. Viviani, A. Kievsky, S. Rosati, and J.E. Beacom, Phys. Rev. C63, 015801 (2001).

[19] J.N. Bahcall, hep-ex/0002018, J.N. Bahcall and P.I. Krastev, Phys. Lett. B436, 243 (1998).

[20] T.-S. Park, K. Kubodera, D.-P. Min, and M. Rho, Nucl. Phys. A684, 101 (2001); see also nucl-th/9904053.

[21] S. Weinberg, Phys. Lett. B251, 288 (1990); Nucl. Phys. B363, 3 (1991); Phys. Lett. B295, 114 (1992).

[22] T.-S. Park, K. Kubodera, D.-P. Min, and M. Rho, Phys. Lett. B472, 232 (2000).

[23] S.R. Beane, V. Bernard, T.-S.H. Lee, and U.-G. Meißner, Phys. Rev. C57, 424 (1998); S.R. Beane, V. Bernard, T.S.H. Lee, U.-G. Meißner, and U. van Kolck, Nucl. Phys. A618, 381 (1997).

[24] T.-S. Park, K. Kubodera, D.-P. Min, and M. Rho, Astrophys. J. 507, 443 (1998).

[25] R. Schiavilla et al., Phys. Rev. C58, 1263 (1998).

[26] V. Bernard, N. Kaiser and U.-G. Meißner, Nucl. Phys. A615, 483 (1997).

[27] T. D. Cohen, J. L. Friar, G. A. Miller, and U. van Kolck, Phys. Rev. C53, 2661 (1996).

[28] D.E. Groom et al. (Particle Data Group), Eur. Phys.
Jour. C15, 1 (2000).

[29] V. Bernard, N. Kaiser, and U.-G. Meißner, Nucl. Phys. B457, 147 (1995).

[30] L.E. Marcucci, R. Schiavilla, M. Viviani, A. Kievsky, and S. Rosati, Phys. Rev. Lett. 84, 5959 (2000).

[31] M. Viviani, A. Kievsky, and S. Rosati, Few-Body Syst. 18, 25 (1995).

[32] M. Viviani, S. Rosati, and A. Kievsky, Phys. Rev. Lett. 81, 1580 (1998).

[33] R.B. Wiringa, V.G.J. Stoks, and R. Schiavilla, Phys. Rev. C51, 38 (1995).

[34] B.S. Pudliner, V.R. Pandharipande, J. Carlson, and R.B. Wiringa, Phys. Rev. Lett. 74, 4396 (1995).

[35] M. Kamionkowski and J.N. Bahcall, Astrophys. J. 420, 884 (1994).

[36] J.C. Hardy, I.S. Towner, V.T. Koslowsky, E. Hagberg, and H. Schmeing, Nucl. Phys. A509, 429 (1990).

[37] S. Fukuda et al., Phys. Rev. Lett. 86, 5651 (2001).

[38] J.N. Bahcall, M.H. Pinsonneault, and S. Basu, Astrophys. J. 555, 990 (2001); astro-ph/0010346.

[39] T.-S. Park, K. Kubodera, D.-P. Min, and M. Rho, unpublished.

[40] J.L. Forest, V.R. Pandharipande, S.C. Pieper, R.B. Wiringa, R. Schiavilla, and A. Arriaga, Phys. Rev. C54, 646 (1996).

[41] J.-W. Chen, G. Rupak, and M.J. Savage, Phys. Lett. B464, 1 (1999).

[42] T.-S. Park, K. Kubodera, D.-P. Min, and M. Rho, Phys. Rev. C58, 637 (1998).

[43] S.R. Beane, P.F. Bedaque, M.J. Savage, and U. van Kolck, Nucl. Phys. A700, 377 (2002); nucl-th/0104030.

[44] S. Bogner, T.T.S. Kuo, L. Coraggio, A. Covello and N. Itaco, "A new approach to shell model effective interactions based on low-momentum nucleon-nucleon potential", nucl-th/0108040.

[45] D.B. Kaplan, M.J. Savage, and M.B. Wise, Phys. Lett. B424, 390 (1998); Nucl. Phys. B534, 329 (1998).

[46] S.R. Beane, P.F. Bedaque, W.C. Haxton, D.R. Phillips and M.J. Savage, in "At the Frontier of Particle Physics Handbook of QCD", ed. by M. Shifman (World Scientific, Singapore, 2001), Vol. 1, p.133

[47] X. Kong and F. Ravendal, Nucl. Phys. A656, 421 (1999); Nucl. Phys. A665, 137 (2000); Phys. Lett. B470, 1 (1999); nucl-th/0004038; M. Butler and J.-W. Chen, nucl-th/0101017.

[48] P. F. Bedaque, H.W. Griesshammer, H.-W. Hammer, and G. Rupak, "Low energy expansion in the three body system to all orders and the triton channel", nuclth/0207034

[49] E. Epelbaum, W. Glöckle, and U.-G. Meißner, Nucl. Phys. A671, 295 (2000); Phys. Rev. Lett. 86, 4787 (2000); E. Epelbaum et al., nucl-th/0109065, and references therein.

[50] T.-S. Park, H. Jung, and D.-P. Min, Phys. Lett. B409, 26 (1997).

[51] N. Fettes, U.-G. Meißner, and S. Steinberg, Nucl. Phys. A 640, 199 (1998).

[52] M. Chemtob and M. Rho, Nucl. Phys. A163, 1 (1971).

[53] I.S. Towner, Phys. Rep. 155, 263 (1987).

[54] E. Ivanov and E. Truhlik, Nucl. Phys. A316, 451 (1979); ibid 437 . 
[55] D.R. Phillips and T.D. Cohen, Nucl. Phys. A668, 45 (2000). 